Prepared for the U.S. Department of Energy

under Contract DE-AC05-76RL01830

\title{
Detection of Actinides via Nuclear Isomer De-Excitation
}

\section{Thesis for MS in Nuclear Engineering Oregon State University}

Christopher J Francy

July 2009

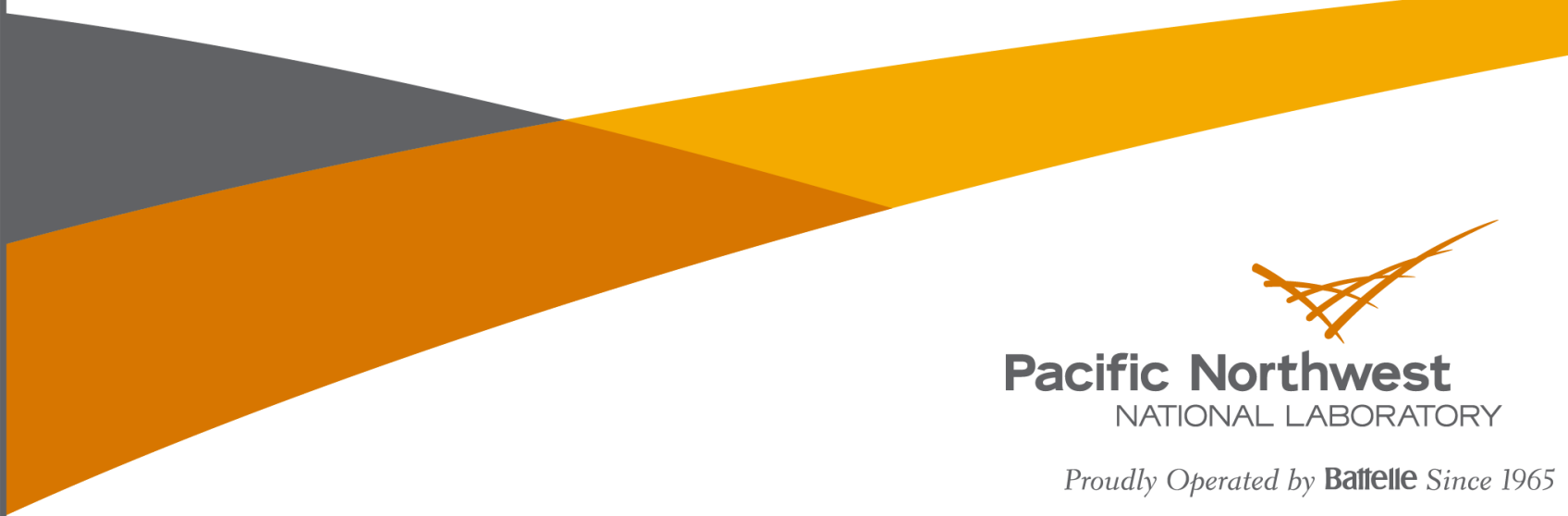




\title{
DISCLAIMER
}

This report was prepared as an account of work sponsored by an agency of the United States Government. Neither the United States Government nor any agency thereof, nor Battelle Memorial Institute, nor any of their employees, makes any warranty, express or implied, or assumes any legal liability or responsibility for the accuracy, completeness, or usefulness of any information, apparatus, product, or process diselosed, or represents that its use would not infringe privately owned rights. Reference herein to any specific commercial product, process, or service by trade name, trademark, manufacturer, or otherwise does not necessarily constitute or imply its endorsement, recommendation, or favoring by the United States Government or any agency thereof, or Battelle Memorial Institute. The views and opinions of authors expressed herein do not necessarily state or reflect those of the United States Government or any agency thereof

\author{
PACIFIC NORTHWEST NATIONAL LABORATORY \\ operated by \\ BATTELLE \\ for the \\ UNITED STATES DEPARTMENT OF ENERGY \\ under Contract DE-AC05-76RL01830 \\ Printed in the United States of America \\ Available to DOE and DOE contractors from the \\ Office of Scientific and Technical Information, \\ P.O. Box 62, Oak Ridge, TN 37831-0062; \\ ph: (865) 576-8401 \\ fax: (865) 576-5728 \\ email: reports@adonis.osti.gov \\ Available to the public from the National Technical Information Service, \\ U.S. Department of Commerce, 5285 Port Royal Rd., Springfield, VA 22161 \\ ph: (800) 553-6847$$
\text { fax: }(703) 605-6900
$$ \\ email: orders@ntis.fedworld.gov \\ online ordering: http://www.ntis.gov/ordering.htm
}

This document was printed on recycled paper.

(9/2003) 


\title{
Detection of Actinides via Nuclear Isomer De-Excitation
}

\author{
Christopher J Francy
}

July 2009

Prepared for

the U.S. Department of Energy

under Contract DE-AC05-76RL01830

Pacific Northwest National Laboratory

Richland, Washington 99352 



\begin{abstract}
Christopher J. Francy for the degree of Master of Science in Nuclear Engineering presented on July 8, 2009.

Title: Detection of Actinides via Nuclear Isomer De-Excitation
\end{abstract}

Abstract approved:

Todd S. Palmer

The detection of illicit weapons-grade nuclear material is a significant technical challenge receiving a great deal of attention in the nuclear community. A proposed solution is to actively bombard the material under investigation with neutrons, populating nuclear isomers in any potential fissile material. These isomers decay quickly, releasing gamma rays that have a higher energy than those from natural radioactive decay, significantly increasing the probability of detection.

This research covers an experiment related to the Actinide Isomer Identification project (AID). The AID project is the investigation of an active interrogation technique that utilizes nuclear isomer production, with the goal of assisting in the interdiction of illicit nuclear materials. In an attempt to find and characterize isomers belonging to ${ }^{235} \mathrm{U}$ and its fission fragments, a ${ }^{232} \mathrm{Th}$ target was bombarded with a monoenergetic ${ }^{6} \mathrm{Li}$ ion beam, operating at $45 \mathrm{MeV}$. 
The experimental data was gathered using an array of silicon, high purity germanium (HPGe) and bismuth germanate (BGO) radiation detectors. The silicon detectors were used to detect reaction products, which were used to start a clock to time gamma ray interactions in the HPGe detectors. The BGO detectors were used to filter out Compton scatter events and background signals.

The data was processed with the spectroscopic software gf3m to identify the signals originating from isomers. The analysis led to the identification and characterization of 19 isomers, of which several were recognized as useful to the goals of the AID project. 


\section{Acknowledgments}

I would like to offer Dr. Jennifer J. Ressler my sincere gratitude, for without her this research would not have been possible. From bringing me onto her team, arranging the funding that supported this work, revising my documents, to answering countless questions, she has been immensely helpful.

Equally deserving of special recognition is Dr. Todd S. Palmer, who has served as my adviser for the past five years. Dr. Palmer has given me the inspiration, guidance, motivation, and professional advice I needed in order to be successful both academically and professionally.

I would also like to recognize Dr. David M. Hamby, whose engaging teaching style and course materials were invaluable to the research performed for this thesis.

Last but certainly not least, I want to offer a big thank you to the members of my committee, who offered their time to evaluate my work. 



\section{Contents}

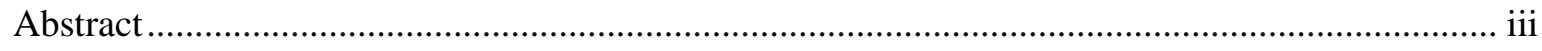

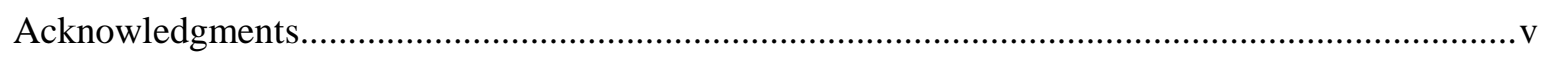

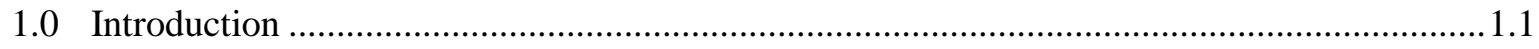

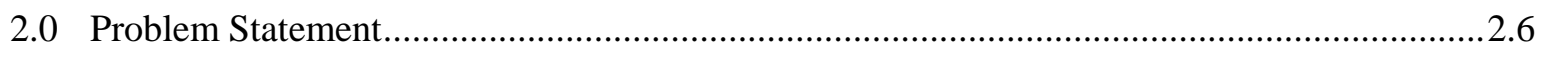

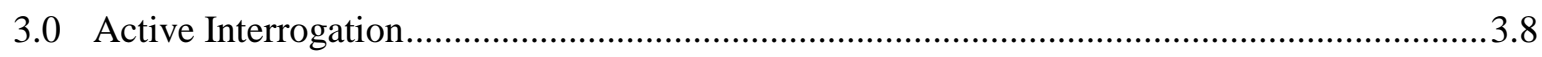

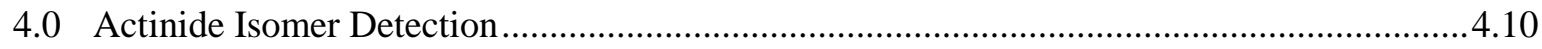

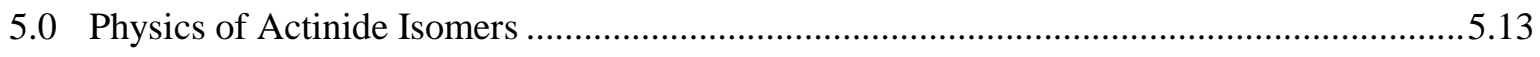

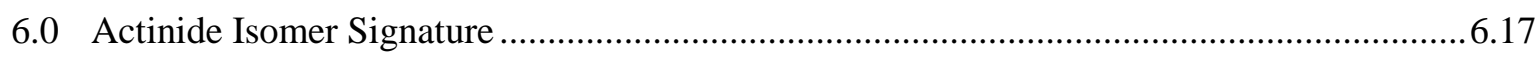

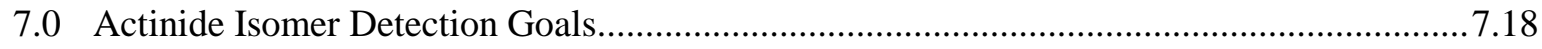

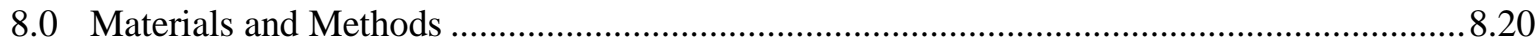

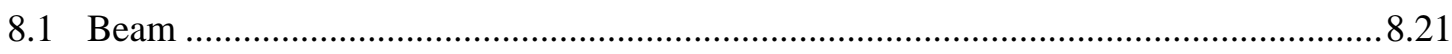

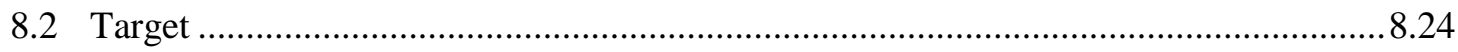

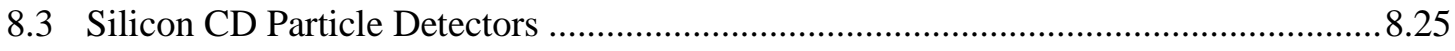

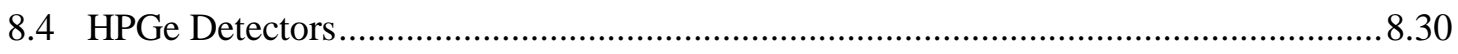

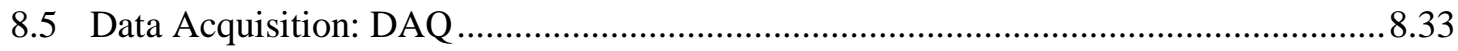

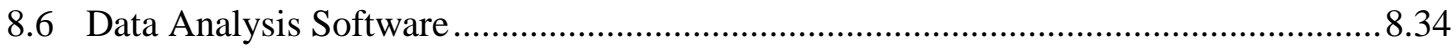

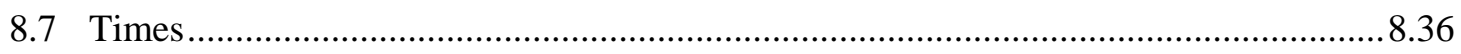

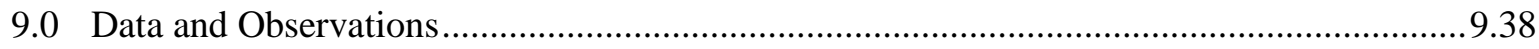

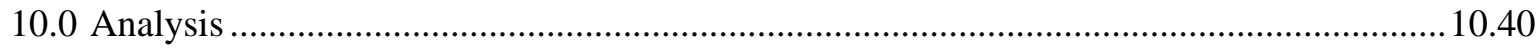

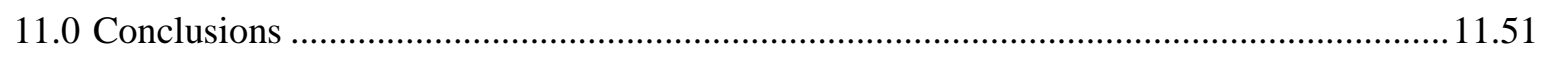

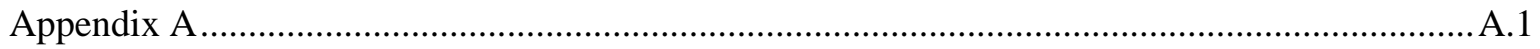




\section{Figures}

Figure 1. A cargo container is placed near a neutron beam and neutron detectors. 3.9

Figure 2. Neutron - gamma spectroscopy system

Figure 3. Energy vs. Deformation with potential wells 5.16

Figure 4. Calculated properties of $\mathrm{U}, \mathrm{Np}$ and $\mathrm{Pu}$ isotopes shape isomers 5.17

Figure 5. Representation of the beam waveform, showing $30 \mu$ s on and $150 \mu$ s off

Figure 6. Ion source before cyclotron injection. 8.23

Figure 7. Photograph of the target chamber (1) and the beam dump (2). 8.25

Figure 8. Sector segmentation on the silicon fission detector. 8.26

Figure 9. Silicon detectors and target wheel loaded in the target chamber. 8.27

Figure 10. $\Delta \mathrm{E}$ vs. $\mathrm{E} 1$ plot showing particle identification in the ${ }^{6} \mathrm{Li}+{ }^{232} \mathrm{Th}$ reaction

Figure 11. Target chamber (1) with germanium detectors in place.

Figure 12. LEPS detector. 8.32

Figure 13. A portion of the detector electronics 8.34

Figure 14. Screenshot of gate created on a peak of interest in gf3m 8.35

Figure 15. Peaks coincident with the gate shown in Figure 14. 8.36

Figure 16. Counts assigned to the TAC and DAQ clocks 8.38

Figure 17. Small sample of the HPGe detector calibration trends

Figure 18. Y-axis projection of the gamma-gamma coincidence matrix in gf $3 \mathrm{~m}$ 10.41

Figure 19. Theoretical fission fragment distribution 10.44

Figure 20. Experimental fission fragment distribution 10.45

Figure 21. Isomeric decays in ${ }^{134} \mathrm{Xe}$ 10.47

Figure 22. Gamma ray energies within $60-100 \mu$ s and 5-45 $\mu$ s of detecting a particle 10.48

Figure 23. Decays recorded within $45 \mu$ s of a particle being detected 10.49 


\section{Tables}

Table 1. Spontaneous fission rates in SNM [2] ..................................................................

Table 2. Known isomers in uranium and plutonium [18] ....................................................14

Table 3. $\mathrm{U}$ and Pu shape isomers ................................................................................17

Table 4. Measured and calculated data for select second-well isomers ............................7.19

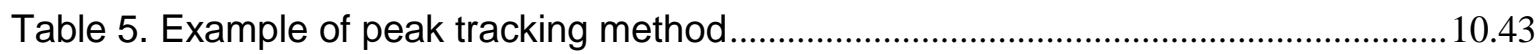

Table 6. Peaks identified from the spectrum shown in Figure 23....................................10.49 



\subsection{Introduction}

With the threat of global terrorism increasing, more attention is being given to the challenges associated with stopping the proliferation of nuclear materials [1]. This endeavor presents a large technical challenge due to the fact that there is a wide variety of potentially threatening radionuclides in existence [1]. This is further complicated by the fact that no one method will successfully detect all known radionuclides; different classes of materials will require different methods depending on what kind of radiation they emit (i.e. gammas vs. neutrons) [2].

The wide varieties of existing radionuclides are often categorized by the way they are weaponized. The first group of radionuclides contains those used in radiological dispersion devices (dirty bombs). These devices utilize the radiation emitted by the chosen radioisotope to cause damage to living tissue by ionizing radiation, and relying on the public's fear of radiation, cause widespread panic and economic destabilization [3]. The second group of radionuclides contains those used in a nuclear device: a device that releases energy by fissioning the nuclei of unstable radioisotopes. These nuclides are found in traditional nuclear weapons, and are known as Special Nuclear Material (SNM) [4]. Detecting the presence of isotopes in this second category is the focus of this research.

Special Nuclear Material is defined by Title I of the Atomic Energy Act of

1954 as plutonium, ${ }^{233} \mathrm{U}$, or uranium enriched in the isotopes ${ }^{233} \mathrm{U}$ or ${ }^{235} \mathrm{U} .{ }^{233} \mathrm{U}$ and plutonium do not occur naturally; however, they can be created in nuclear 
reactors. ${ }^{233} \mathrm{U}$ is made in reactors using thorium as fuel, and plutonium is created in reactors that use ${ }^{235} \mathrm{U}$ and ${ }^{238} \mathrm{U}$ as fuel. Enriched uranium is produced in an enrichment facility that uses technology such as gaseous diffusion or high-speed centrifuges [4].

The most common approaches for detecting SNM utilize radiation detectors. The basic principle of a radiation detector is that the radiation enters the detector and its energy is converted into an electrical signal, amplified and then sent to hardware that counts the number of events [5]. The most basic detectors operate in a binary fashion - they indicate whether radiation is present or not. If a scientist or law enforcement officer detects radiation using one of these simple devices, they have no way or knowing if it is Special Nuclear Material. The sample would need to be isolated and then sent to a laboratory for further analysis.

However, recent advances in radiation detection have produced devices that permit spectroscopy to be performed in the field. Spectroscopy involves looking at the energy distribution, or spectrum, associated with the radiation emitted from the sample [6]. Spectroscopy relies on the fact that some forms of radiation, especially gamma rays, are emitted from discrete energy levels in the nucleus as radionuclides decay to lower energy levels [7]. Furthermore, most radionuclides have unique decay energies, allowing relatively easy identification of the decaying radionuclide. 
Spectroscopy can be used to monitor and aid the flow of materials through a security checkpoint. For example, a spectroscopic device would allow a Customs and Border Patrol (CBP) agent to assess the threat of an unknown material without having to immediately send samples to CBP's analysis group known as Laboratories and Scientific Services (LSS). This is important because spending time investigating benign radioactive materials is detrimental to the US economy [8].

While it is important to maintain a stable U.S. economy, it is also vitally important to detect and stop illicit Special Nuclear Material from entering the United States. The consequences of failing to interdict special nuclear material justify the significant investment made in technologies for its detection [3]. Should a nuclear weapon be detonated by terrorists on U.S. soil, there would be a large number of deaths and illnesses, and unprecedented economic damage and demoralization of the United States' population. The difficulty of acquiring SNM is the most significant barrier to the development of an atomic bomb [9]. It is imperative to stop the trafficking of these materials because, as noted in an Office of Technology Assessment study, once the SNM has been acquired construction of a nuclear bomb could be within the capabilities of a sophisticated group of state actors or terrorists [10]. 
Detection of SNM is very difficult, as the passive radiation emitted in normal radioactive decay is dominated by alpha particles. Alpha particles are readily absorbed in surrounding material, and are therefore not easily detected.

Heavier actinides will undergo spontaneous fission, emitting neutrons. The number of neutrons emitted can be estimated as:

$$
R_{n}=m \cdot \lambda \cdot\left(\frac{N_{A}}{A}\right) \cdot S F_{B R} \cdot \bar{v}
$$

given the mass $(m)$, decay constant $(\lambda)$, Avogadro's number $\left(N_{A}=6.02 \times 10^{23}\right)$, atomic mass $(\mathrm{A})$ in $\mathrm{g} / \mathrm{mol}$, spontaneous fission branching ratio $\left(\mathrm{SF}_{\mathrm{BR}}\right)$ and average number of neutrons emitted $(\bar{v})$. Table 1 shows that the primary isotopes of interest $\left({ }^{235} \mathrm{U}\right.$ and $\left.{ }^{239} \mathrm{Pu}\right)$ have very low neutron emission rates, making passive detection difficult.

Table 1. Spontaneous fission rates in SNM [2].

\begin{tabular}{|c|c|c|c|c|}
\hline Nuclide & Half-life & Fission prob. per decay (\%) & Neutrons per fission & Neutrons per (gram · second) \\
\hline${ }^{235} \mathrm{U}$ & $7.04 \times 10^{8}$ years & $7.0 \times 10^{-9} \%$ & 1.86 & $1.0 \times 10^{-5}$ \\
\hline${ }^{235} \mathrm{U}$ & $4.47 \times 10^{9}$ years & $5.4 \times 10^{-5} \%$ & 2.07 & 0.0136 \\
\hline${ }^{239} \mathrm{Pu}$ & $2.41 \times 10^{4}$ years & $4.4 \times 10^{-10} \%$ & 2.16 & $2.2 \times 10^{-2}$ \\
\hline${ }^{240} \mathrm{Pu}$ & 6569 years & $5.0 \times 10^{-5} \%$ & 2.21 & 920 \\
\hline
\end{tabular}

Photons will be emitted in the alpha and spontaneous fission decays. However, a major hurdle in detecting SNM is its relatively weak gamma flux [11]. In a vacuum, radiation disperses from a point source as a function of the inverse distance squared,

$$
\phi \propto \frac{1}{r^{2}}
$$


where $\phi$ is the scalar flux and $r$ is the distance from the source. Therefore, the radiation signal can be very weak even at short distances.

This is further compounded by the fact that these weak gammas are low energy, and therefore easy to shield. The intensity of a radiation signal also experiences an exponential decay through an absorbing medium,

$$
I=I_{0} e^{-\mu_{\ell} x}
$$

where $I$ and $I_{0}$ are the observed and emitted intensities, $x$ is the sample thickness and $\mu_{\ell}$ is the linear mass attenuation coefficient. The linear mass attenuation coefficient is dependent on the gamma energy and sample material. Lower gamma energies, such as those emitted by the actinides, have larger values of $\mu_{\ell}$. Lighter elements have much smaller $\mu_{\ell}$ values than the heavy actinides. For example, a $186-\mathrm{keV}$ photon from the decay of ${ }^{235} \mathrm{U}$ has a $\mu_{\ell}$ of approximately $0.33 \mathrm{~cm}^{-1}$ in aluminum, but about $27.49 \mathrm{~cm}^{-1}$ in uranium metal $[6,12]$.

In this thesis, a potential solution to the problems associated with the detection of the SNM actinides is explored. The sample is interrogated with a pulsed beam of neutrons, causing the actinides to populate relatively long-lived (isomeric) nuclear excited states. These states will decay between the neutron pulses, emitting high energy gamma-rays. The gamma energies are expected to be much higher than those emitted by standard radioactive decay. These higher energy photons will be able to penetrate through significantly more shielding. 
The challenge associated with this technique is that the isomeric states have not been observed in all actinide isotopes, nor have the gamma decays been observed. This work discusses experiments to search for the actinide isomer states, fission fragment isomer states, and a possible signature for SNM detection.

\subsection{Problem Statement}

There is a significant need for new technologies to aid in the search for illicit Special Nuclear Material (SNM) [1]. The main vectors for smuggling illicit goods are sea, air, rail and land border crossings. At these ports of entry, billions of dollars worth of commercial goods pass into the United States each month, and almost ten million vehicles cross the borders [13]. Because it is such a colossal task to screen every entering vehicle or piece of cargo, radiation detectors have been installed at these locations to support the Department of Homeland Security mission. These detectors are primarily polyvinyl toluene (PVT) scintillators which provide the "yes or no" system response - no spectroscopy is being preformed.

In addition to the polyvinyl toluene detectors, sodium iodide (Nal) detectors capable of spectroscopy are being brought into use. These detectors have the advantage of letting the operator know what radionuclides are present 
in the load of cargo. Sodium iodide detectors provide some spectroscopic information, and the detected spectra are compared with those contained within a software library. If the cargo contains naturally occurring radioactive material (NORM), the shipment is allowed to pass through. If the cargo contains a potentially illegitimate shipment of radioactive materials, or the software is unable to match the spectra to those contained within the library, the cargo is flagged for further investigation.

While the polyvinyl toluene and sodium iodide detector systems excel at detecting many isotopes used in radiological dispersal devices, challenges remain with respect to the detection of Special Nuclear Material. Since radiological dispersal devices utilize the radioactive properties of the chosen material, elements emitting high energy photons are optimal. High-energy gamma rays are difficult to shield and easy to detect. Unfortunately with SNM, some of the radionuclides of concern emit very low energy gamma rays which are easy to conceal $[5,11]$. In many circumstances, such as with ${ }^{235} \mathrm{U}$, the self shielding properties of the uranium combined with the other legitimate cargo in a container (or other surrounding materials) will be enough to prevent the detection of these emitted radiations. This raises a concern as one of the most threatening radionuclides is also one of the easiest to shield.

Because of the properties of ${ }^{235} \mathrm{U}$ and a few other select isotopes, a better method is needed to detect them. The current PVT and Nal detectors operate on 
the principle of passive detection; "passive" refers to the fact that these detectors do not cause any changes to their environment or surroundings.

\subsection{Active Interrogation}

Another method of detecting materials is through active interrogation. With active interrogation, the detector is coupled with an emitted signal that actively interacts with the item being screened rather than passively accepting signals. An active detector would be analogous to an airport X-ray machine. To detect contraband, the X-ray machine releases photons which strike the target, and information is gleaned based on the photon interactions in the scanned object. This technique is commonly employed because only a small fraction of the scanned contraband yields a signal that can be sensed using passive detection methods. This active interrogation principle can be applied to the search for radioactive materials.

One active interrogation technology used to detect radioactive materials involves bombarding a nucleus with photons or particles, and causing further photons and/or particles to be released [7]. The emitted radiation can then be detected using the same methods used for passive detection. A popular approach for active interrogation is to use a pulsed neutron beam to irradiate the sample. The neutrons will induce fission in the actinides, releasing more neutrons. For example, a single neutron inducing fission on a ${ }^{239} \mathrm{Pu}$ atom will 
release 2.874 neutrons, on average [14]. A small fraction of these secondary neutrons will be delayed relative to the interrogating neutron pulse; observation of delayed neutrons suggests the presence of SNM.

A schematic for an active interrogation system using neutrons is shown in Figure 1 below.

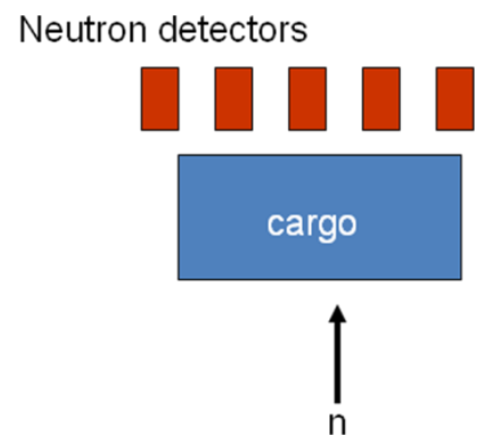

Figure 1. A cargo container is placed near a neutron beam and neutron detectors.

There are important challenges involved with neutron interrogation. Interrogating neutrons can slowly lose energy, reflecting from material under investigation as well as the surrounding walls and structures of the scanning facility. These neutrons may then be detected during the delayed (beam off) time while the detector is not actively emitting particles. The number of fission neutrons is quite low, so the background must be controlled and/or understood well. Additionally, some light ions will be excited and undergo a reaction which will cause a neutron to be ejected. 
Neutrons are difficult to detect with commercial systems, as most detectors have low detection efficiencies. Most commercial detectors are made of hydrogenous materials due to the higher interaction probability for neutrons with low-Z elements. However, this also means that neutrons are easily moderated in hydrogenous and organic material (e.g. paper, wood, etc) hindering detection.

Observations of photons provides a much more distinct signature.

Photons are usually emitted with discrete energy distributions, meaning that spectroscopic analysis may be utilized to characterize the origin of the particles [5]. During the neutron pulse, an enormous flux of gamma rays will be released. The flux between pulses is significantly less. There is ongoing research to look for high energy gamma-rays emitted in the beta decay of fission fragments; one such process is being investigated at Lawrence Livermore National Laboratory [15]. Another example would be Nuclear Resonance Fluorescence, which is being investigated at Pacific Northwest National Laboratory [16] and Lawrence Livermore National Laboratory [17].

Actinide Isomer Detection (AID) is a similar concept, although the timescale is shortened, reducing interferences from background. Beta decay is a "slow" process, with half-lives greater than milliseconds. The isomers sought in AID decay on the microsecond timescale.

\subsection{Actinide Isomer Detection}

One approach to active interrogation of SNM is the creation of nuclear isomers of the nuclide(s) of interest. A nuclear isomer differs from a chemical 
isomer. In chemistry, an isomer refers to molecules with the same formula yet different structures. A nuclear isomer is a metastable state of the nucleus [7]. When the nucleus is struck by a projectile, energy is imparted to it, and it is raised above the ground state. If the nucleus decays back to the ground state (releases its energy) very quickly, it is considered a prompt gamma emission. If an excited state remains populated for an extended period of time $\left(10^{-6}\right.$ seconds or longer), it is classified as an isomer [7]. These emitted photons are potentially detectable and useful for identifying SNM.

An example of active interrogation utilizing isomer detection involves deeply penetrating particles, such as neutrons, being delivered to a cargo container. Neutrons are released in a pulse; a burst of the particles is released in a short amount of time $(\sim 10 \mu \mathrm{s})$, and then the beam is stopped for an extended period ( 20 ms). During the pulse, neutrons pass through shielding suitable for gamma radiation (i.e. lead vs. borated polyethylene), and strike nuclei of special nuclear material. Upon striking a nucleus, an isomer may be populated. The isomer then decays back down to a lower energy level, releasing a high-energy gamma. This photon is released during the time period when the neutron beam is turned off. This released photon exits the material, penetrates the shielding, and makes it back to the detector. The signal processing software associated with the detector performs spectroscopic analysis on the measured photon spectrum. Once the algorithm matches the energy to that of a known SNM 
isomer, the detector alerts the operator to the presence of special nuclear material.

Alternatively, a particle (such as a neutron) may strike the nucleus and cause fission events, which will then populate the sample with fission fragments. These fission fragments may also be analyzed for gamma rays due to isomeric transition.

AID is complementary to currently proposed neutron interrogation scenarios in that the gamma-ray detectors can be added to the neutron detectors (as shown in Figure 2) to make an improved spectroscopy system.

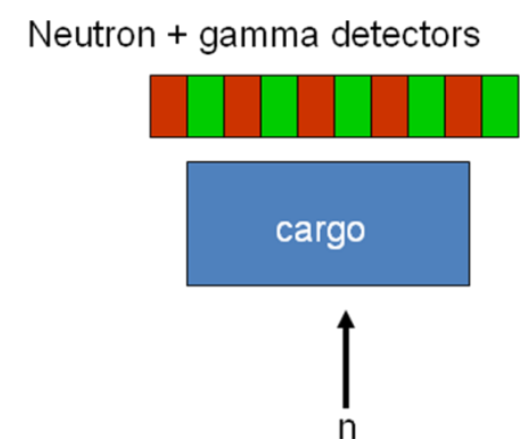

Figure 2. Neutron - gamma spectroscopy system

One potential threat to the development of this technology is that many actinide (SNM) isomers are only theorized to exist; they have not yet been measured and documented. To successfully employ this technology in the more advanced search for illicit special nuclear materials, many experiments must be performed. First, and most importantly, the isomers must be 1) shown to exist and 2) characterized. Characterization involves measurements of decay energies 
and half-lives of the isomeric states. Once the characterization is complete, experiments to examine the isomeric population in an interrogating (i.e. neutron) beam must be performed. Results from the experimental studies are then used in numerical simulations to evaluate the feasibility and/or optimize the design of the AID system. This thesis describes an experiment designed to address the existence and properties of actinide and fission fragment isomers, and their characterizations.

\subsection{Physics of Actinide Isomers}

In addition to aiding national security projects, this research adds to the body of knowledge in the area of fundamental nuclear physics through the characterization of new isomers. There are already many known isomeric states in the actinide isotopes. Table 2 highlights isomers in the uranium and plutonium isotopes. 
Table 2. Known isomers in uranium and plutonium [18]. $\Delta$ l represents the change in spin angular momentum between the ground and isomeric states

\begin{tabular}{|c|c|c|c|}
\hline Isotope & $\mathbf{T}_{1 / 2}$ & $\boldsymbol{\Delta l}$ & Gamma Energy [keV] \\
\hline${ }^{234} \mathrm{U}$ & $33 \mu \mathrm{s}$ & 6 & $899,227,153,100,44$ \\
\hline${ }^{236} \mathrm{U}$ & $120 \mathrm{~ns}$ & 0 & $2062,1783,967,642,279$ \\
\hline${ }^{238} \mathrm{U}$ & $280 \mathrm{~ns}$ & 0 & $2557,2514,1879,635,45$ \\
\hline${ }^{239} \mathrm{U}$ & $780 \mathrm{~ns}$ & 2 & 134 \\
\hline${ }^{237} \mathrm{Pu}$ & $180 \mathrm{~ms}$ & 0 & 146 \\
\hline${ }^{239} \mathrm{Pu}$ & $193 \mathrm{~ns}$ & 3 & $278,106,8$ \\
\hline${ }^{239} \mathrm{Pu}$ & $7.5 \mu \mathrm{s}$ & -2 & $?$ \\
\hline${ }^{240} \mathrm{Pu}$ & $165 \mathrm{~ns}$ & 5 & $601,566,448,153,99$ \\
\hline${ }^{241} \mathrm{Pu}$ & $880 \mathrm{~ns}$ & 2 & 162 \\
\hline${ }^{241} \mathrm{Pu}$ & $21 \mu \mathrm{s}$ & $?$ & $?$ \\
\hline
\end{tabular}

To be used for AID, the isomers must have the following characteristics:

- $\quad$ Half-life $\sim 1-100 \mu \mathrm{s}$

- One or more gamma decay energies $>1000 \mathrm{keV}$

- $\quad$ Be populated by the interrogation beam

Known half-lives and gamma decay energies are shown in Table 2. Also shown is the change in spin angular momentum $(\Delta \mathrm{l})$ from the ground to the isomeric state; to be populated by a neutron interrogation beam, $\Delta \mathrm{l}$ should be only 1 or 2 .

The isomers that would fit best with the proposed AID technique exist in ${ }^{236} \mathrm{U},{ }^{238} \mathrm{U}$, and ${ }^{239} \mathrm{Pu}$. These are all so-called "shape isomers". 
Previous research on actinide isomers includes observation of shape isomers in ${ }^{236} U$ [19] and ${ }^{238} U$ [20], as well as other actinides such as neptunium, plutonium, americium, curium and berkelium [20]. These states are thought to occur when the nucleus is in a greatly deformed state. The premise behind a deformed nuclear state is that as energy is imparted to the nucleus, vibration increases and the shape becomes distorted, as the overall volume is incompressible. As the vibrational energy increases, the deformation increases, with discrete potential wells in which the nucleus may remain temporarily [7]. If the deformation continues to increase in selected nuclides, fission will occur. Figure 3 illustrates this process and shows that it takes a discrete amount of additional energy to continue the deformation process. In these deformed states it is possible that the nucleus may stay in a potential well temporarily. If the deformation energy increases beyond the well - fission will occur, whereas if it cannot overcome the energy barrier of the well, the excess energy will be released as a discrete gamma ray and the nucleus will return to the ground state [21]. 


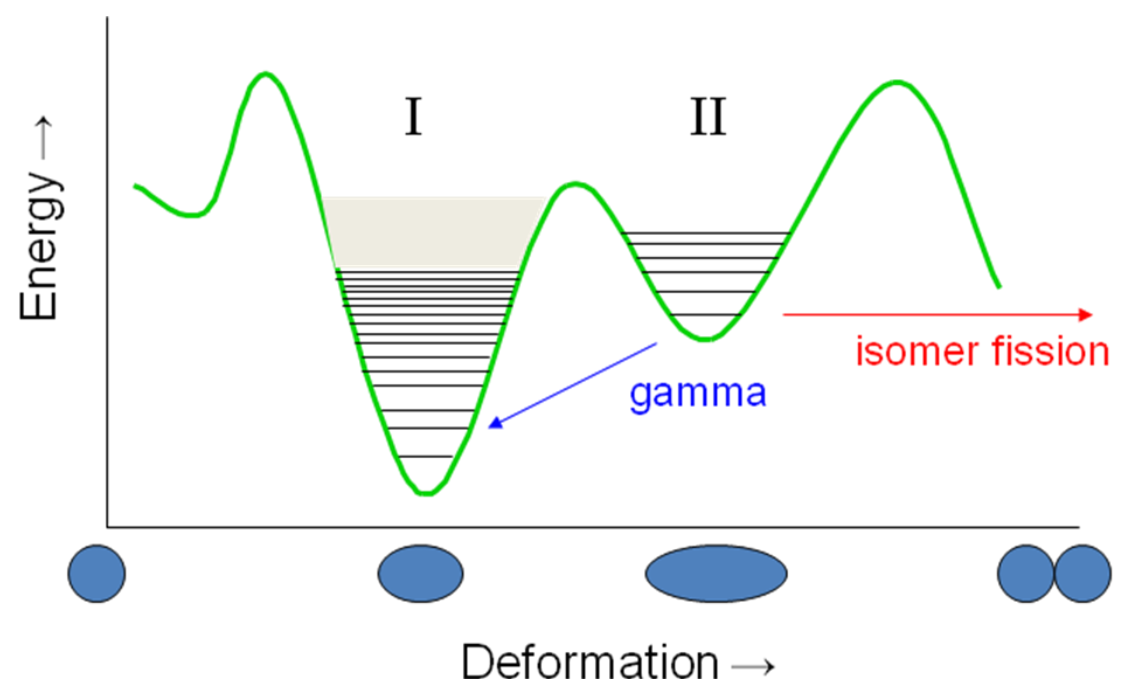

Figure 3. Energy vs. Deformation with potential wells

Shape isomers have been observed at $2-3 \mathrm{MeV}$ excitation energy in a number of plutonium and americium isotopes, and are also expected to be present in uranium and neptunium. Shape isomers have attracted some attention due to the high probability for fission decay in the heavier elements. However, few studies have attempted to measure the gamma decay branch from the isomeric states. The lighter isotopes, notably uranium and neptunium, are expected to have significant ( $\geq 90 \%$ ) gamma decay branches near $2 \mathrm{MeV}[20]$. Calculated isomeric properties for a range of uranium, neptunium and plutonium isotopes are shown in Figure 4. Few experimental comparisons are available; characteristics for known shape isomers are compared to predicted values in Table 3.

It is clear from these comparisons that the theory predicts the isomer properties very well. One striking example is ${ }^{238} \mathrm{U}$, with an isomer at $2.6 \mathrm{MeV}$ excitation energy. This state depopulates with a half-life of $280 \mathrm{~ns}$ and the emission of a $2.5 \mathrm{MeV}$ Y-ray (67\%) or $1.9 \mathrm{MeV}$ Y-ray (26\%). The probability for 
fission (5\%) and internal conversion $(<1 \%)$ are much less. The isomeric state has been populated and measured in neutron bombardment reactions at low energy $(<5 \mathrm{MeV})[20,22-25]$.

\begin{tabular}{|c|c|c|c|c|c|c|c|c|c|}
\hline $\begin{array}{c}{ }^{236} \mathrm{Pu} \\
\mathrm{T}_{1 / 2} 0.2 \mathrm{~ns} \\
\mathrm{E}^{*} 2.7 \mathrm{MeV} \\
\mathrm{F}(\gamma)--\end{array}$ & $\begin{array}{c}{ }^{237} \mathrm{Pu} \\
\mathrm{T}_{1 / 2} 2.6 \mu \mathrm{s} \\
\mathrm{E}^{*} 2.6 \mathrm{MeV} \\
\mathrm{F}(\gamma) 0.8 \%\end{array}$ & $\begin{array}{c}{ }^{238} \mathrm{Pu} \\
\mathrm{T}_{1 / 2} 1.0 \mathrm{~ns} \\
\mathrm{E}^{*} 2.4 \mathrm{MeV} \\
\mathrm{F}(\gamma)--\end{array}$ & $\begin{array}{c}{ }^{239} \mathrm{Pu} \\
\mathrm{T}_{1 / 2} 17 \mu \mathrm{s} \\
\mathrm{E}^{*} 2.6 \mathrm{MeV} \\
\mathrm{F}(\gamma) 4 \%\end{array}$ & $\begin{array}{c}{ }^{240} \mathrm{Pu} \\
\mathrm{T}_{1 / 2} 2.7 \mathrm{~ns} \\
\mathrm{E}^{*} 2.3 \mathrm{MeV} \\
\mathrm{F}(\gamma)--\end{array}$ & $\begin{array}{c}{ }^{241} \mathrm{Pu} \\
\mathrm{T}_{1 / 2} 38 \mu \mathrm{s} \\
\mathrm{E}^{*} 2.3 \mathrm{MeV} \\
\mathrm{F}(v) 14 \%\end{array}$ & $\begin{array}{c}{ }^{242} \mathrm{Pu} \\
\mathrm{T}_{1 / 2} 3.7 \mathrm{~ns} \\
\mathrm{E}^{*} 2.4 \mathrm{MeV} \\
\mathrm{F}(\gamma) 0.1 \%\end{array}$ & $\begin{array}{c}{ }^{243} \mathrm{Pu} \\
\mathrm{T}_{1 / 2} 28 \mu \mathrm{s} \\
\mathrm{E}^{*} 2.6 \mathrm{MeV} \\
\mathrm{F}(\gamma) 31 \%\end{array}$ & $\begin{array}{c}{ }^{244} \mathrm{Pu} \\
\mathrm{T}_{1 / 2} 2.4 \mathrm{~ns} \\
\mathrm{E}^{*} 2.3 \mathrm{MeV} \\
\mathrm{F}(\gamma) 0.2 \%\end{array}$ & $\begin{array}{c}{ }^{245} \mathrm{Pu} \\
\mathrm{T}_{1 / 2} 6.7 \mu \mathrm{s} \\
\mathrm{E}^{*} 2.3 \mathrm{MeV} \\
\mathrm{F}(\gamma) 48 \%\end{array}$ \\
\hline & & $\begin{array}{c}{ }^{237} \mathrm{~Np} \\
\mathrm{~T}_{1 / 2} 53 \mu \mathrm{s} \\
\mathrm{E}^{*} 2.4 \mathrm{MeV} \\
\mathrm{F}(\gamma) 90 \%\end{array}$ & $\begin{array}{c}{ }^{238} \mathrm{~Np} \\
\mathrm{~T}_{1 / 2} 2.2 \mathrm{~s} \\
\mathrm{E}^{*} 2.6 \mathrm{MeV} \\
\mathrm{F}(\gamma) 96 \%\end{array}$ & $\begin{array}{c}{ }^{239} \mathrm{~Np} \\
\mathrm{~T}_{1 / 2} 55 \mu \mathrm{s} \\
\mathrm{E}^{*} 2.2 \mathrm{MeV} \\
\mathrm{F}(\gamma) 97 \%\end{array}$ & $\begin{array}{c}240 \mathrm{~Np} \\
\mathrm{~T}_{1 / 2} 1.3 \mathrm{~s} \\
\mathrm{E}^{*} 2.2 \mathrm{MeV} \\
\mathrm{F}(\gamma) 99 \%\end{array}$ & & & & \\
\hline $\begin{array}{c}234 \mathrm{U} \\
\mathrm{T}_{1 / 2} 54 \mathrm{~ns} \\
\mathrm{E}^{*} 2.7 \mathrm{MeV} \\
\mathrm{F}(\gamma) 34 \%\end{array}$ & $\begin{array}{c}{ }^{235} \mathrm{U} \\
\mathrm{T}_{1 / 2} 6.9 \mu \mathrm{s} \\
\mathrm{E}^{*} 2.6 \mathrm{MeV} \\
\mathrm{F}(\mathrm{v}) 100 \%\end{array}$ & $\begin{array}{c}{ }^{236} \mathrm{U} \\
\mathrm{T}_{1 / 2} 170 \mathrm{~ns} \\
\mathrm{E}^{*} 2.4 \mathrm{MeV} \\
\mathrm{F}(\gamma) 62 \%\end{array}$ & $\begin{array}{c}{ }^{237} \mathrm{U} \\
\mathrm{T}_{1 / 2} 9.4 \mu \mathrm{s} \\
\mathrm{E}^{*} 2.6 \mathrm{MeV} \\
\mathrm{F}(\gamma) 100 \%\end{array}$ & $\begin{array}{c}{ }^{238} \mathrm{U} \\
\mathrm{T}_{1 / 2} 230 \mathrm{~ns} \\
\mathrm{E}^{*} 2.3 \mathrm{MeV} \\
\mathrm{F}(\gamma) 81 \%\end{array}$ & $\begin{array}{c}{ }^{239} \mathrm{U} \\
\mathrm{T}_{1 / 2} 6.4 \mu \mathrm{s} \\
\mathrm{E}^{*} 2.3 \mathrm{MeV} \\
\mathrm{F}(\mathrm{v}) 100 \%\end{array}$ & & & & \\
\hline
\end{tabular}

Figure 4. Calculated properties of $\mathrm{U}, \mathrm{Np}$ and $\mathrm{Pu}$ isotopes shape isomers. These were derived from Weigmann \& Theobald's paper on Evaluation of Fission Barrier Parameters [20]. The theoretical decay half-life $\left(T_{1 / 2}\right)$, excitation energy $\left(E^{*}\right)$, and gamma branch $(F(\gamma))$ are shown.

Only fission and gamma decay were considered in the calculation.

Table 3. $U$ and Pu shape isomers. Comparison between theoretical [20] and experimental [26-30] properties illustrate the strong predictive power of the calculations. Theoretical and experimental half-lives $\left(T_{1 / 2}\right)$, excitation energies $\left(E^{*}\right)$ and gamma branches $(F(\gamma))$ are shown with the observed gamma emission energy (EY) and relative intensities (ly). Note that many $U$ and $\mathrm{Np}$ isotopes have not been studied.

\begin{tabular}{|c|c|c|c|c|c|c|c|}
\hline & \multicolumn{3}{|c|}{ Theory } & \multicolumn{3}{c|}{ Experiment } \\
\cline { 2 - 8 } & $\mathbf{T}_{\mathbf{1} / \mathbf{2}}$ & $\mathbf{E}^{*}$ & $\mathbf{F}(\gamma)$ & $\mathbf{T}_{\mathbf{1} / \mathbf{2}}$ & $\mathbf{E}^{*}$ & $\mathbf{F}(\gamma)$ & $\mathbf{E} \gamma(\mathbf{I} \gamma)$ \\
\hline${ }^{236} \mathrm{U}$ & $170 \mathrm{~ns}$ & $2.4 \mathrm{MeV}$ & $62 \%$ & $120 \mathrm{~ns}$ & $2.75 \mathrm{MeV}$ & $87 \%$ & $\begin{array}{c}2.71(<12), 2.06(26), 1.78(100), \\
1.17(20), 0.56(12) \mathrm{MeV}\end{array}$ \\
\hline${ }^{238} \mathrm{U}$ & $230 \mathrm{~ns}$ & $2.3 \mathrm{MeV}$ & $100 \%$ & $280 \mathrm{~ns}$ & $2.56 \mathrm{MeV}$ & $97 \%$ & $2.51(100), 1.88(49) \mathrm{MeV}$ \\
\hline${ }^{236} \mathrm{Pu}$ & $0.2 \mathrm{~ns}$ & $2.7 \mathrm{MeV}$ & -- & $0.04 \mathrm{~ns}$ & $\sim 3.0 \mathrm{MeV}$ & $?$ & Gamma decay not studied \\
\hline${ }^{237} \mathrm{Pu}$ & $2.6 \mu \mathrm{s}$ & $2.6 \mathrm{MeV}$ & $0.80 \%$ & $1.1 \mu \mathrm{s}$ & $\sim 2.9 \mathrm{MeV}$ & $?$ & Gamma decay not studied \\
\hline${ }^{238} \mathrm{Pu}$ & $1.0 \mathrm{~ns}$ & $2.4 \mathrm{MeV}$ & -- & $0.6 \mathrm{~ns}$ & $\sim 2.4 \mathrm{MeV}$ & $?$ & Gamma decay not studied \\
\hline${ }^{239} \mathrm{Pu}$ & $17 \mu \mathrm{s}$ & $2.6 \mathrm{MeV}$ & $4 \%$ & $7.5 \mu \mathrm{s}$ & $\sim 3.1 \mathrm{MeV}$ & $\sim 0 \%$ & One photofission study (Ref. [30]) \\
\hline${ }^{240} \mathrm{Pu}$ & $2.7 \mathrm{~ns}$ & $2.3 \mathrm{MeV}$ & -- & $3.7 \mathrm{~ns}$ & $\sim 2.8 \mathrm{MeV}$ & $?$ & Gamma decay not studied \\
\hline${ }^{241} \mathrm{Pu}$ & $38 \mu \mathrm{s}$ & $2.3 \mathrm{MeV}$ & $14 \%$ & $21 \mu \mathrm{s}$ & $\sim 2.2 \mathrm{MeV}$ & $?$ & Gamma decay not studied \\
\hline${ }^{242} \mathrm{Pu}$ & $3.7 \mathrm{~ns}$ & $2.4 \mathrm{MeV}$ & $0.10 \%$ & $3.5 \mathrm{~ns}$ & $\sim 2.2 \mathrm{MeV}$ & $?$ & Gamma decay not studied \\
\hline
\end{tabular}

\subsection{Actinide Isomer Signature}

Photons from the decay of the isomeric state are expected to have high energy, $2 \mathrm{MeV}$. As each isotope will have a different isomer excitation energy 
and gamma decay energy, the high energy photons released in the decay will provide a distinct isotopic signature. In addition, the decay will have a unique half-life, providing a distinctive time signature as well.

Inelastic scattering reactions using fast interrogation neutrons can populate the isomeric state with relatively high cross sections, typically a few barns in the actinide region, making this a potentially sensitive technique. Due to the delayed nature of the isomeric decay, the state will de-populate outside of the incident neutron pulse. The delayed photons can be observed using commercially available high-resolution detection systems.

The observation of discrete high-energy photons with unique time characteristics from particular actinide isotopes or their fission fragments suggests the presence of SNM.

\subsection{Actinide Isomer Detection Goals}

The goal of this research is to determine if isomers of special nuclear material can be observed. However, shape isomers have not been observed in

all actinide isotopes, and gamma decay has only been observed in ${ }^{236} \mathrm{U}$ and ${ }^{238} \mathrm{U}$. The first step in exploring the use of AID as a signature for special nuclear material is to investigate the existence of the isomer and characterize the decay.

Experimentally, understanding the gamma branch is difficult because there is a large background signal from fission, scatter reactions, and natural background radiation. The characterization study must be designed to minimize background and contaminant non-isomeric gamma rays. The detection system 
must be superior, with multiple time, energy, and reaction-type selections possible. The data analysis must also be performed carefully to ensure proper attribution of the observed gammas.

The initial search study within the AID project aimed to identify the predicted isomers in ${ }^{235} \mathrm{U},{ }^{237} \mathrm{~Np}$ and ${ }^{239} \mathrm{Pu}$. Table 4 highlights current data on these isotopes.

Table 4. Measured and calculated data for select second-well isomers. Measured data are from [31] $\left.{ }^{235} \mathrm{U}\right)$, [32] and [33] $\left({ }^{237} \mathrm{~Np}\right)$, and [29] $\left({ }^{239} \mathrm{Pu}\right)$. Calculated data are from Weigmann \& Theobald's work [20]

\begin{tabular}{|c|c|c|c|c|c|}
\hline Isotope & $\mathbf{E}^{*}[\mathrm{keV}]$, meas & $\mathbf{T}_{1 / 2}$, meas & BR $[\gamma]$, meas & $\mathbf{T}_{1 / 2}$, th & BR $[\gamma]$, th \\
\hline${ }^{235} \mathrm{U}$ & -- & $3.8(18) \mathrm{ms}$ & -- & $7 \mu \mathrm{s}$ & $100 \%$ \\
\hline${ }^{237} \mathrm{~Np}$ & $2800(400)$ & $45(5) \mathrm{ns}$ & -- & $50 \mu \mathrm{s}$ & $90 \%$ \\
\hline${ }^{239} \mathrm{Pu}$ & $3100(200)$ & $7.5(10) \mu \mathrm{s}$ & -- & $17 \mu \mathrm{s}$ & $4 \%$ \\
\hline
\end{tabular}

Excited states in these isotopes may be populated by transfer reactions using a light-ion beam (i.e. ${ }^{7} \mathrm{Li},{ }^{9} \mathrm{Be}$ ) on a long-lived actinide target (i.e. ${ }^{232} \mathrm{Th}$, $\left.{ }^{235} \mathrm{U}\right)$. Use of a thin $\left(\sim 500 \mu \mathrm{g} / \mathrm{cm}^{2}\right)$ target will allow transfer products to pass out of the back of the target and into a particle detector array. Knowledge of the transferred particle gives rise to the product nucleus. For example,

$$
{ }^{6} \mathrm{Li}+{ }^{235} \mathrm{U} \rightarrow{ }^{239} \mathrm{Pu}+\mathrm{dn}
$$

Detection of a deuteron suggests that ${ }^{239} \mathrm{Pu}$ was created. A gamma detection array surrounding the target can be used to collect gamma-decay energies and intensities for each isotope populated. Finally, the light-ion beam is pulsed to monitor the gamma-decays when the beam is on ("prompt") and off (“delayed", isomeric). 
While the ultimate goal of AID is to populate the isomers using neutrons, a

${ }^{6} \mathrm{Li}$ beam was used for the characterization phase of the experiment. ${ }^{6} \mathrm{Li}$ was chosen because it has the ability to create ${ }^{235} \mathrm{U}$ by reacting with ${ }^{232} \mathrm{Th}$, and leave it in an excited state. The de-excitation energy can be used to characterize a ${ }^{235} \mathrm{U}$ isomer. By using transfer reactions, the outgoing particle can be easily detected, helping define the product isotope, as described above. By combining highefficiency particle and gamma detectors, timing between isotope creation (via particle detection) and decay (via gamma detection) allows half-lives to be measured. $A^{6} \mathrm{Li}$ beam also has the added benefit of causing fissions; the resulting fission fragments may have isomers useful to the goal of detecting SNM.

This thesis describes the experimental design and data analysis in a search for the isomers of ${ }^{235} \mathrm{U}$ and fission fragments produced in the reaction. Numerous isomers were identified, which may be used to detect the presence of special nuclear material.

\subsection{Materials and Methods}

A complex detection system is necessary to observe the isomeric decays. This system is described in detail below. 


\subsection{Beam}

To discover and characterize the actinide isomers, a facility capable of producing a monoenergetic beam of charged particles is needed. The facility that was chosen for this work was the 88-inch cyclotron at Lawrence Berkeley National Laboratory (LBNL).

The cyclotron was operated at a frequency of $6.12596 \mathrm{MHz}$, which means that the ${ }^{6} \mathrm{Li}$ was delivered in bunches $163.240 \mathrm{~ns}$ apart. To study isomers, the beam needed to be stopped, or blocked, for brief periods of time. A standard pulse generator was used to create the desired square waveform for the beam on/off periods - 30 microseconds set to "on" (low voltage) and 150 microseconds set to "off" (high voltage). This was accomplished by sending the signal to an electrostatic beam deflector which swept the beam away during the off period. To switch the beam on or off took less than one microsecond. The beam deflector was placed between the ion source and the injection point of the cyclotron. The purpose of pulsing the beam is to find the isomers - radiation detected with the beam on is "prompt" whereas radiation detected while the beam is off is of interest. A graphical representation of the beam waveform can be seen in Figure 5. 


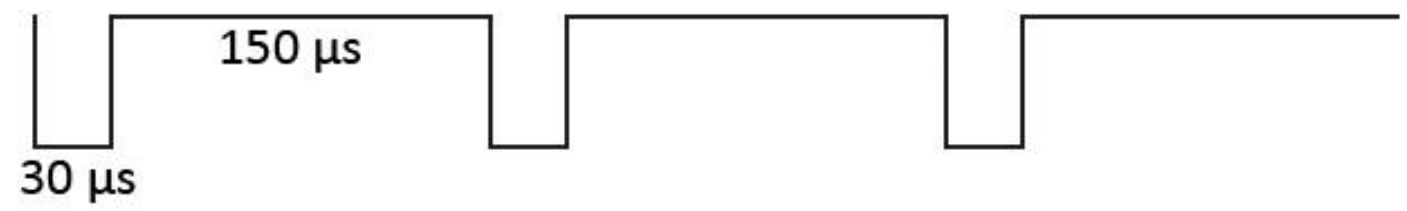

Figure 5. Representation of the beam waveform, showing $30 \mu$ s on and $150 \mu$ s off.

The beam is physically deflected at the ion source, before particles enter the cyclotron. This means the beam ions must continue to travel through the facility to the target once they enter the cyclotron. Since the ions already in the cyclotron will continue to travel to the experimental station, the electronic "beam off" signal must be delayed by the amount of time it takes to empty the cyclotron and have the particles reach the target. The same holds true for beam initiation - the deflector switch opens, the ions travel to the cyclotron which then emits the beam down the line. Therefore, the "beam on" and the "beam off" signals need to be delayed by the time it takes to empty/fill and deliver. This time was measured to be 70 microseconds. To correct for this, the 70 microsecond delay is added to the on/off signal used for the detector trigger electronics, synchronizing the beam signal with the detectors. The cyclotron ion source can be seen in Figure 6. 


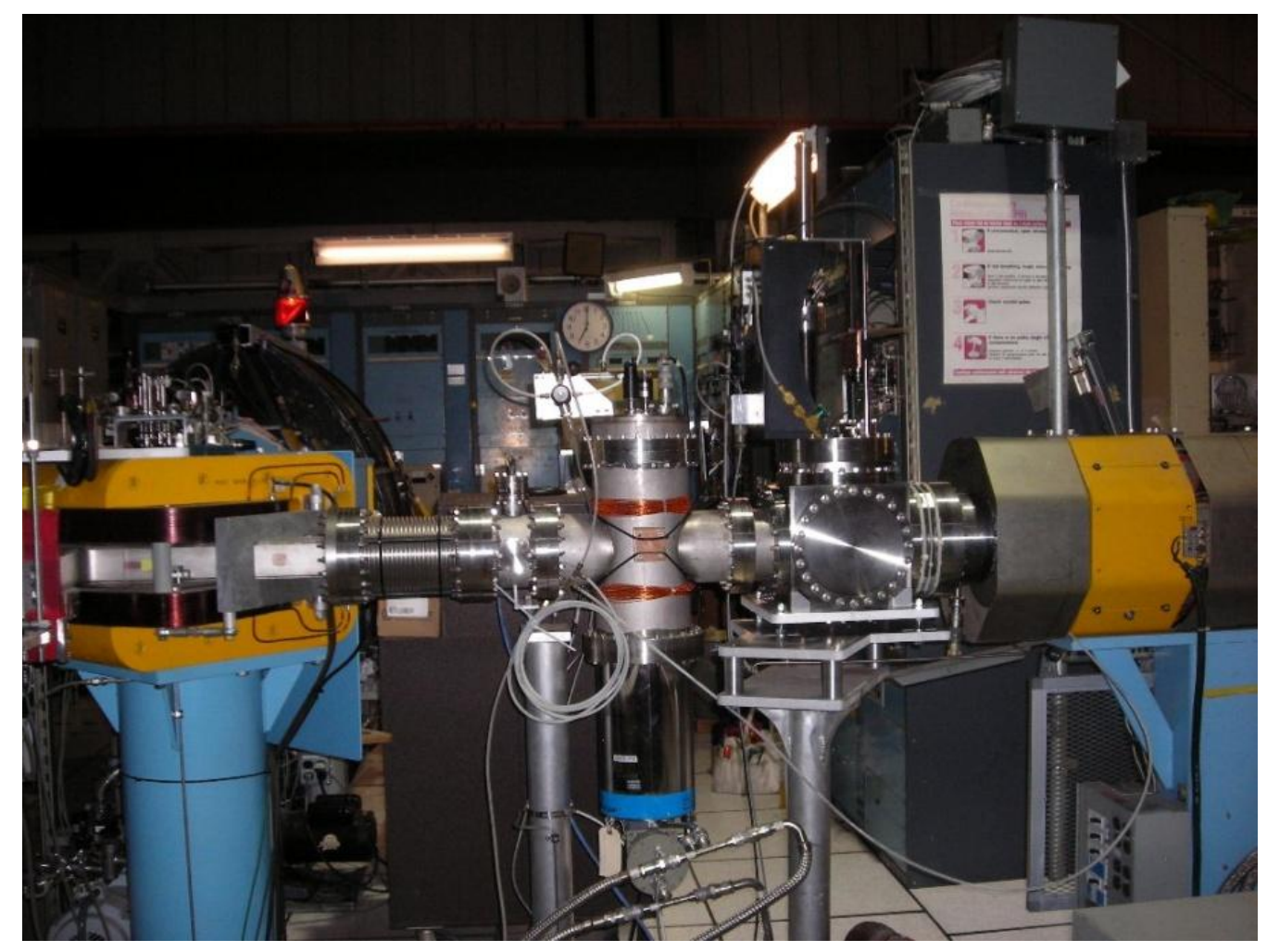

Figure 6. Ion source before cyclotron injection

The beam consisted of ${ }^{6} \mathrm{Li}$ particles ionized to the +2 charge state, and energized to $45 \mathrm{MeV}$. This energy was chosen to maximize the number of isomers created while minimizing the number of fission events. The minimum energy required, for the ${ }^{6} \mathrm{Li}$ ions to overcome the coulomb barrier of the thorium nucleus, is about $36 \mathrm{MeV}$. Using PACE, a software tool that calculates approximate production cross-sections, it was determined that in addition to the $36 \mathrm{MeV}$ to overcome the coulomb barrier, an additional $9 \mathrm{MeV}$ would be needed for optimal production of ${ }^{235} \mathrm{U}$ from ${ }^{6} \mathrm{Li}$ with ${ }^{232} \mathrm{Th}$ [34]. 


\subsection{Target}

The target was a pure foil $889 \mu \mathrm{g} / \mathrm{cm}^{2}$ thick of thorium-232. The foil target was made by J. Greene at Argonne National Lab (IL). A ${ }^{232}$ Th foil was chosen as it produces the following reactions:

- $\quad$ Proton out: ${ }^{232} \mathrm{Th}+{ }^{6} \mathrm{Li} \rightarrow{ }^{237} \mathrm{U}+\mathrm{p}$

- $\quad$ Deuteron out: ${ }^{232} \mathrm{Th}+{ }^{6} \mathrm{Li} \rightarrow{ }^{236} \mathrm{U}+\mathrm{d},{ }^{232} \mathrm{Th}+{ }^{6} \mathrm{Li} \rightarrow{ }^{235} \mathrm{U}+\mathrm{dn}$

- $\quad$ Alpha out: ${ }^{232} \mathrm{Th}+{ }^{6} \mathrm{Li} \rightarrow{ }^{234} \mathrm{~Pa}+\alpha$

Of the listed reactions, the deuteron out reaction is of primary interest.

The thorium target was held in place using a circular wheel that allows several different targets to be loaded into the target chamber. New targets can be placed into the path of the beam by turning a knob rather than opening the chamber, which is kept under vacuum. In addition to the primary target, several collimators were loaded to aid the beam tuning process. The target chamber is shown in Figure 7. 


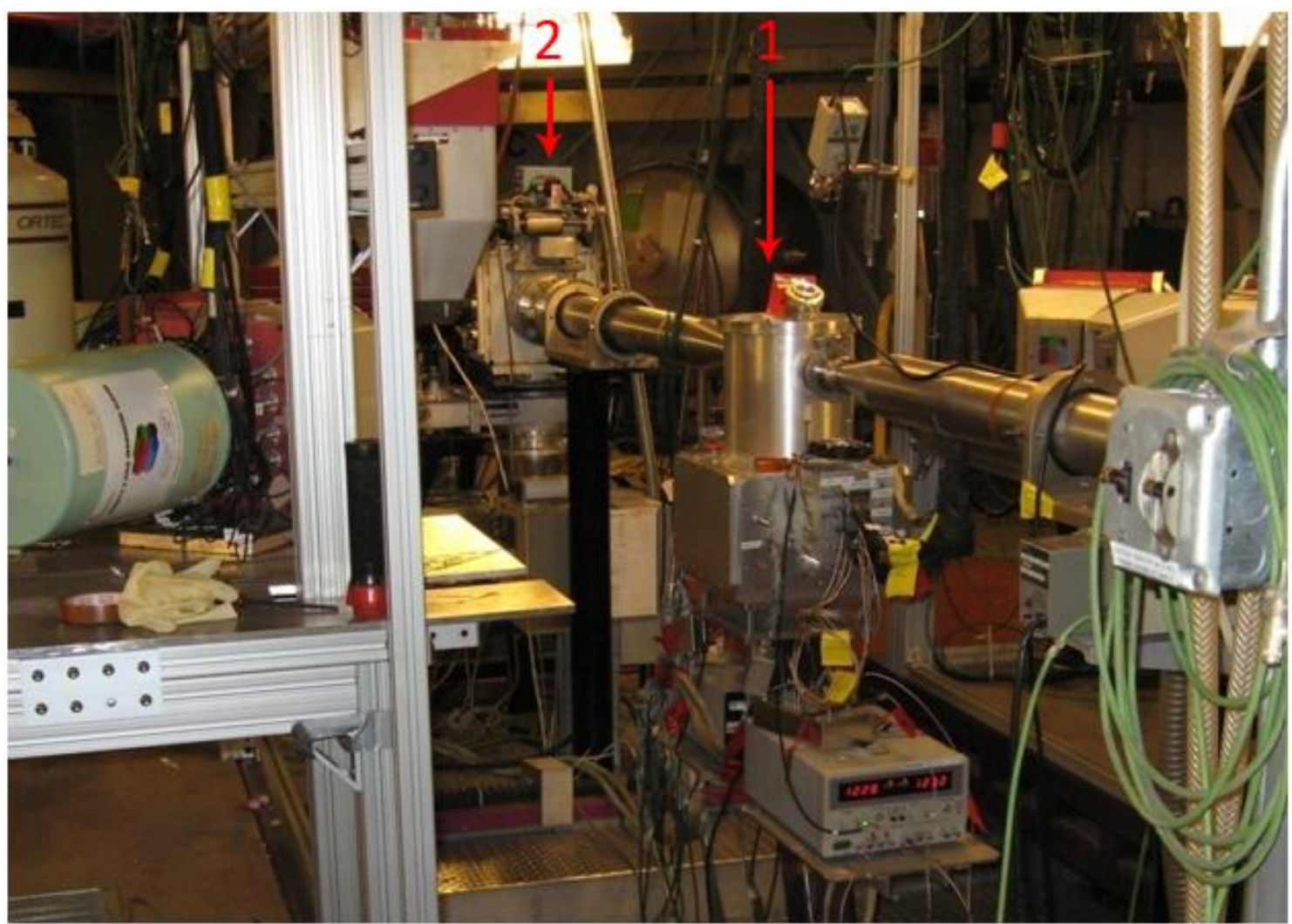

Figure 7. Photograph of the target chamber (1) and the beam dump (2).

\subsection{Silicon CD Particle Detectors}

Directly surrounding the thorium target were four detectors; these detectors are composed of silicon and resemble compact discs. Holes were placed in the center of the detectors so they would not be exposed to the direct flux of the beam, as much of the beam passed through the target without interacting. On one side, the discs were divided into several sectors with each sector being a detection zone, as shown in Figure 8. On the opposite side, the discs were segmented into rings so the particles could be tracked as they moved throughout the system. This allows particles coming from the target to be identified separately from the beam spray upstream from the target. 


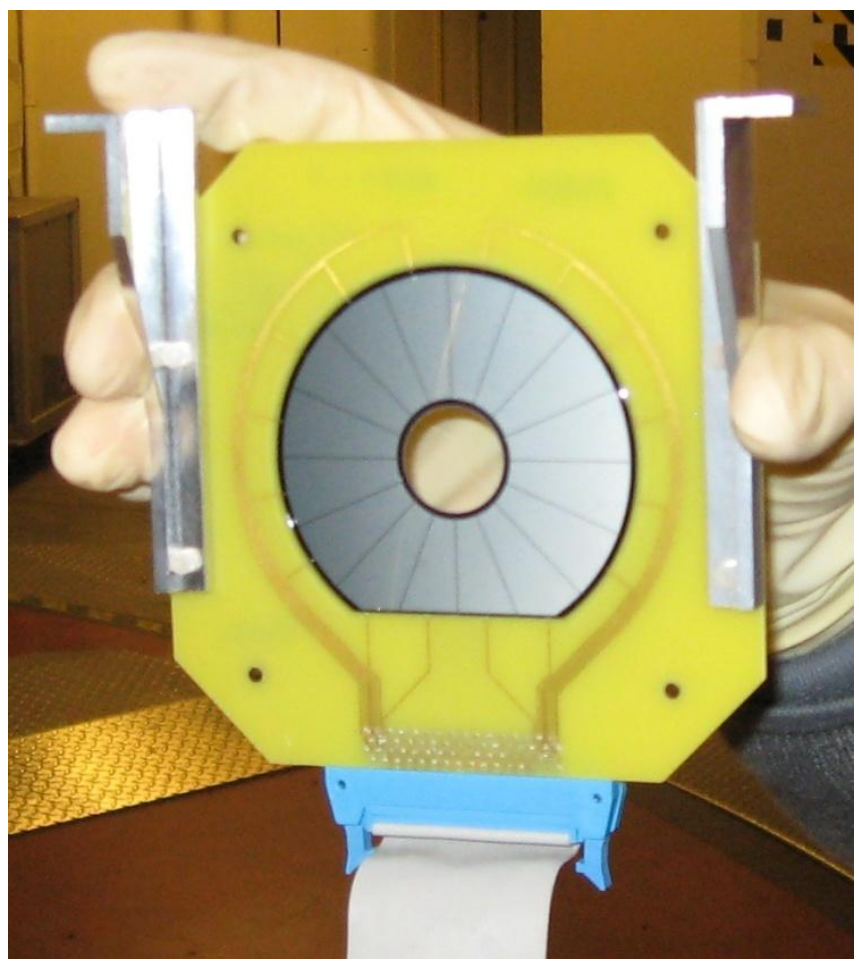

Figure 8. Sector segmentation on the silicon fission detector.

The following detectors were placed directly behind the thorium target:

$\Delta \mathrm{E}$, which was $150 \mu \mathrm{m}$ thick

E1, which was $994 \mu \mathrm{m}$ thick

E2, which was $1001 \mu \mathrm{m}$ thick

The fission detector was placed directly in front of the target, and was 140 $\mu \mathrm{m}$ thick.

In Figure 9, the arrangement of the silicon detectors is shown from above, looking down with the top cover of the reaction chamber removed. The beam 
enters on the right side, and exits out the left side. In the figure the arrows point to the following items:

Silicon fission detector

Rotary target holder

$\Delta \mathrm{E}$ silicon detector

E2 silicon detector

The $\mathrm{E} 1$ detector is in place but is not visible as the aluminum bracket close to $\Delta \mathrm{E}$ is obstructing the view.

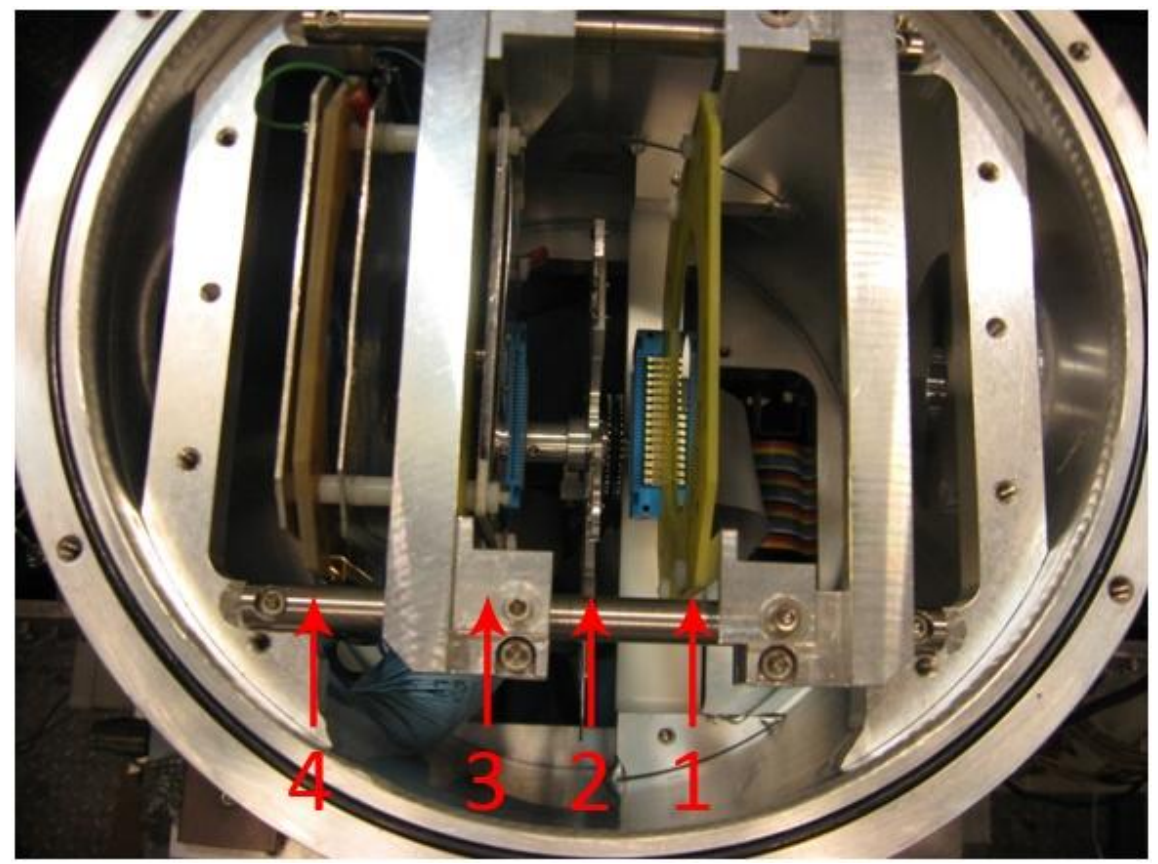

Figure 9. Silicon detectors and target wheel loaded in the target chamber. From right to left are the fission detector (1), the target wheel (2), the $\Delta \mathrm{E}$ detector (3) and the E2 detector (4).

The Si detectors were used for gross counting and discrimination of charged particles from one another (i.e. an alpha vs. deuteron). Particle discrimination is based on which detectors register an interaction. For example, an alpha particle would penetrate the first $(\Delta \mathrm{E})$ and be fully absorbed in the 
middle piece of silicon (E1) whereas deuterons and protons will pass through the middle detector. A stainless steel shield was used before the first detector to shield it from scattered lithium ions from the beam. Aluminum shields with $a+300$ $\mathrm{V}$ bias were placed between E1 and E2 and after E2 to deflect electrons away from the silicon detectors. This is necessary due to the fact that there was enough voltage applied to the silicon detectors to deplete them. Too many unwanted electrons striking the silicon would cause the detectors to no longer be depleted, thereby greatly reducing their ability to detect particles.

These detectors are vital, as particle interactions start the clock for decay time measurements. 


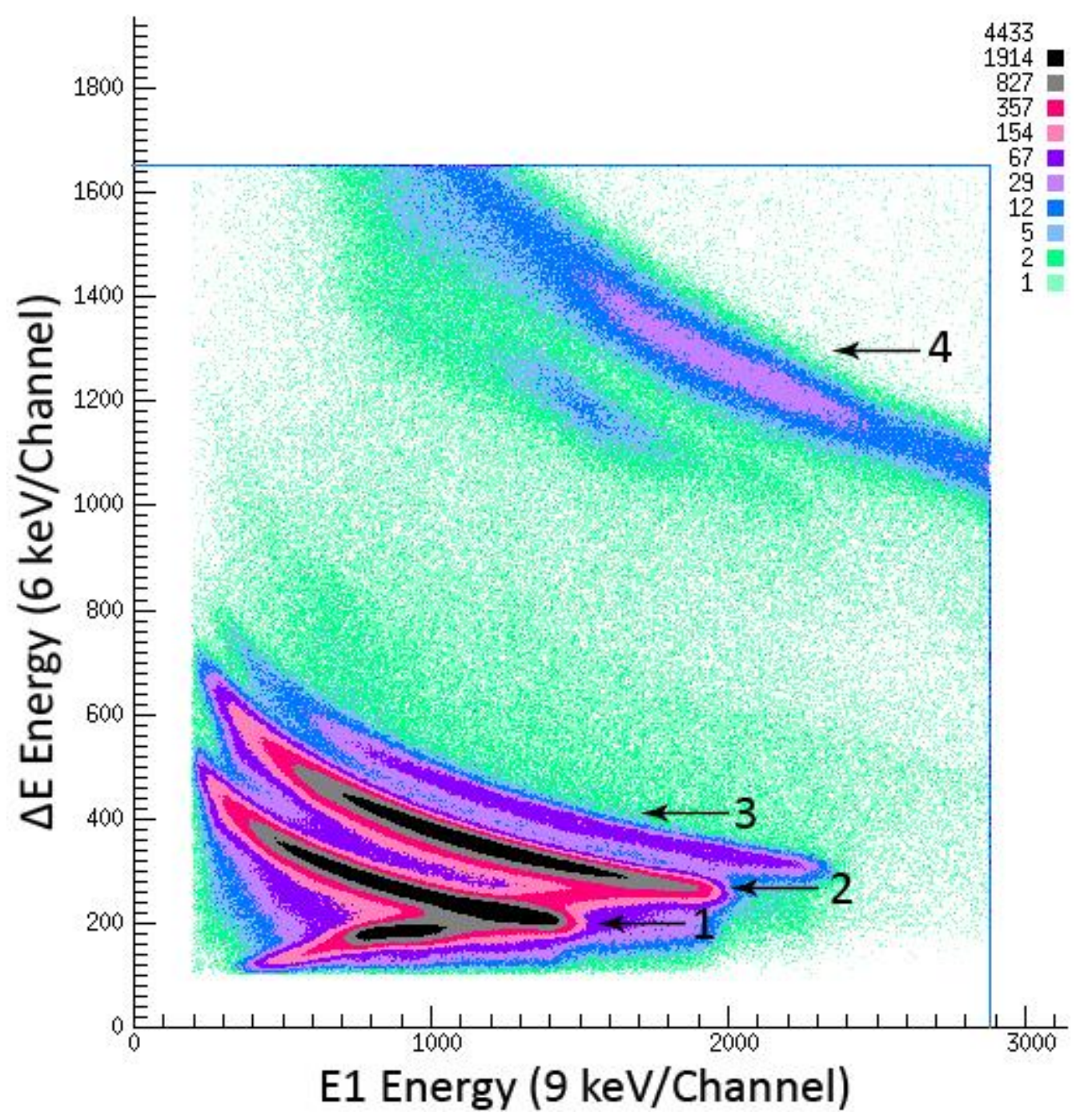

Figure 10. $\Delta \mathrm{E}$ vs. $\mathrm{E} 1$ plot showing particle identification in the ${ }^{6} \mathrm{Li}+{ }^{232} \mathrm{Th}$ reaction. Shown are protons (1), deuterons (2), tritons (3) and alphas (4).

The fission, $\Delta \mathrm{E}$, and $\mathrm{E} 1$ detectors have 32 rings on one side and 16 sectors on the other for particle tracking. Each channel was paired so there were effectively 16 rings and 8 sectors on each detector. The rings have higher resolution than the sectors, and faced the target when possible. For E1, the sectors faced the target because it was desirable to put $\Delta \mathrm{E}$ and $\mathrm{E} 1$ as close together as possible so the coincidence between the two detectors is maximized. E2 has a wider opening in the center, to adjust for the fact that recoils from the 
target will have a larger change in direction relative to the beam. The E2 detector had 16 rings and 16 sectors.

\subsection{HPGe Detectors}

Surrounding the target area were six Canberra clover detectors, five of which were operating properly. The detectors are referred to as clovers because each unit is made of four smaller germanium crystals placed together so an "addback" can be performed. An add-back is when a gamma scatters out of one crystal into another, and the sum of energy deposited can be determined. Clovers are useful for determining the direction of the emitted photon; however, for this experiment that information was not required.

Each clover was surrounded by a BGO (bismuth germanate) scintillator to reject gamma rays that scatter out of the detector altogether (Compton scatter events), as well as background radiation that scatters into the detector. Additionally, each clover has a tungsten shield on the detector system to prevent gamma rays from entering at unwanted angles. This system is depicted in Figure 11.

The detector that was not properly operating had a failed BGO rejection system. The data collected from it was not useless; however it was contaminated with a much larger background signal. 


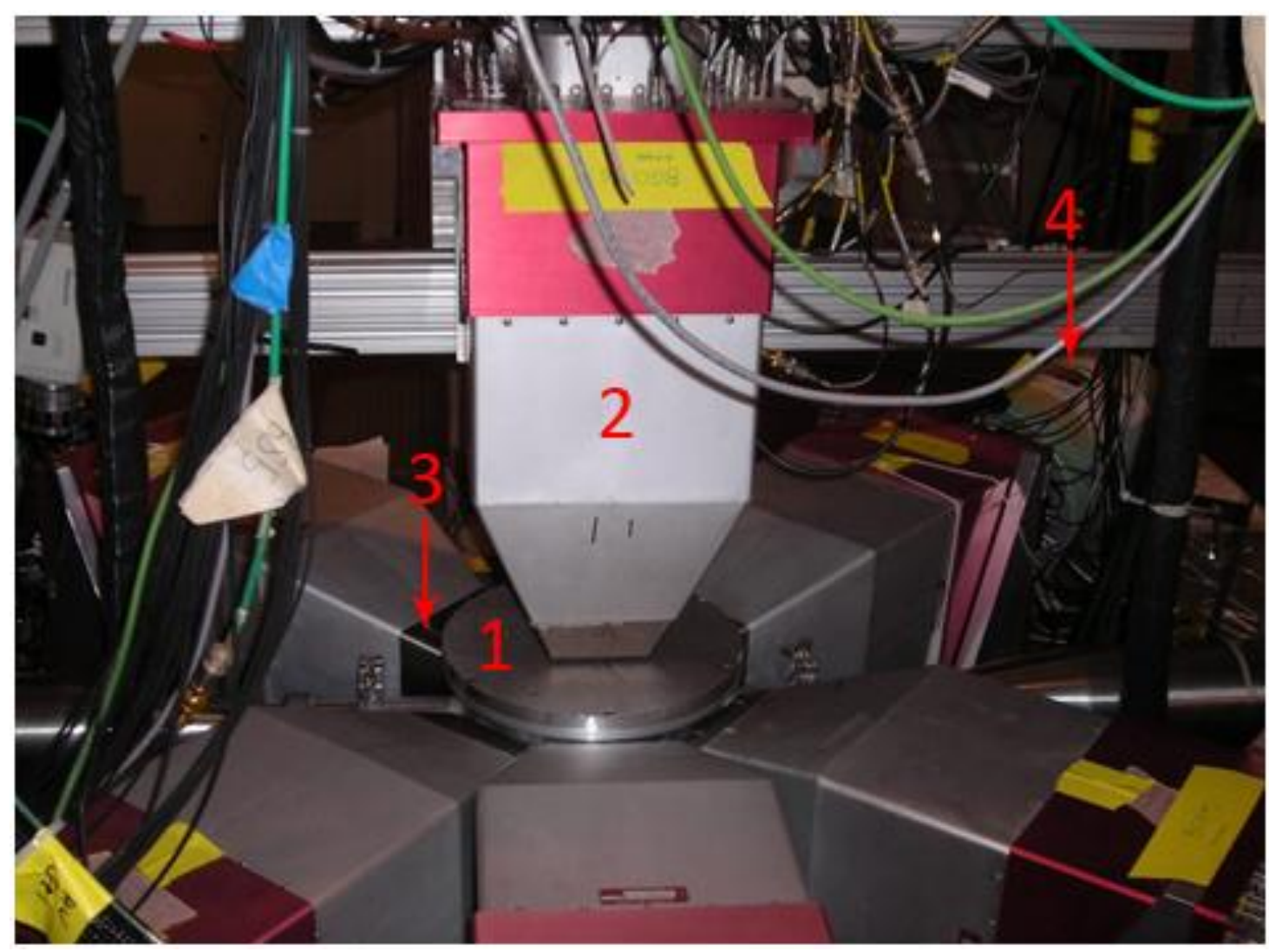

Figure 11. Target chamber (1) with germanium detectors in place. The BGO detectors surrounding the germanium detectors can be seen (2), as well as the tungsten shields (3). One of the liquid nitrogen dewars for the germanium detectors is pictured as well (4).

In addition to the germanium/BGO detectors surrounding the target, there was one ORTEC Low Energy Photon Spectrometer (LEPS) to measure x-rays and low energy gamma rays that were produced. This is pictured in Figure 12. 


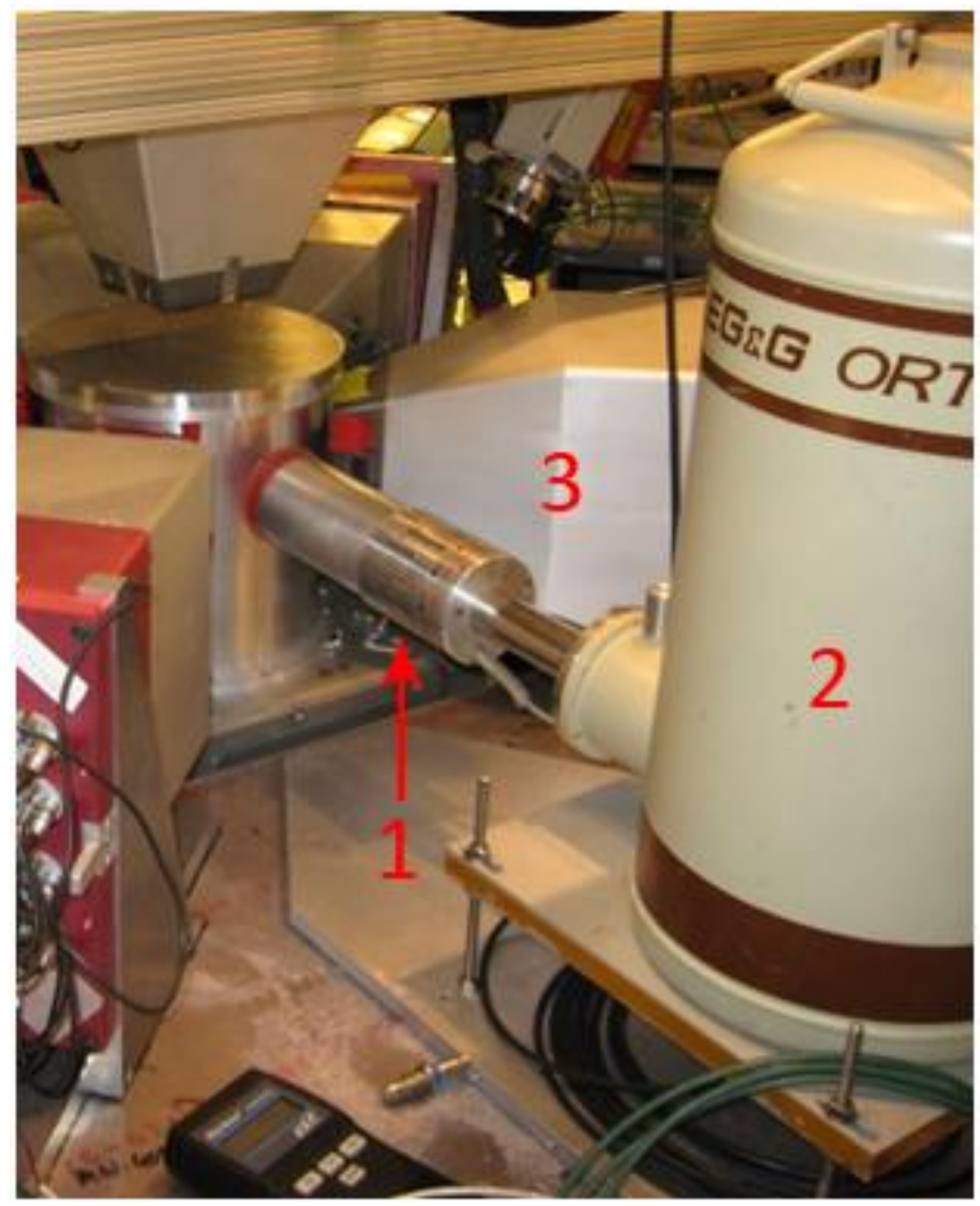

Figure 12. LEPS detector. The LEPS detector is pictured (1) with its liquid nitrogen dewar (2) and the germanium clover detectors (3).

These detectors were used to measure the gamma rays emitted by the isomers under investigation. A large number of detectors are needed because the isomer production rate is expected to be small, resulting in a very low fluence seen by the detectors. As a result, many detectors were placed as close to the sample as possible (see Figure 11). 


\subsection{Data Acquisition: DAQ}

The data acquisition system is "trigger" driven. The trigger simply tells the data acquisition to start recording data. A "gate" opens for a short time, and if a detector has energy data, it will be written out to computer disk.

Two triggers were used, and either could start the data acquisition:

Trigger 1: Particle detected

Trigger 2: Beam off signal + (gamma or fission)

Each trigger would open the gate for certain event types. If the gate was closed, data would not be recorded for that detector type regardless of the presence of a signal. Particles, fission, and gamma events each had separate gates:

Particle gate: Trigger 1 only

Fission gate: Trigger 1 or Trigger 2

Gamma gate: Trigger 2 only

The data acquisition was a complex system; a portion of the electronic modules and cables used for this experiment are shown in Figure 13. 


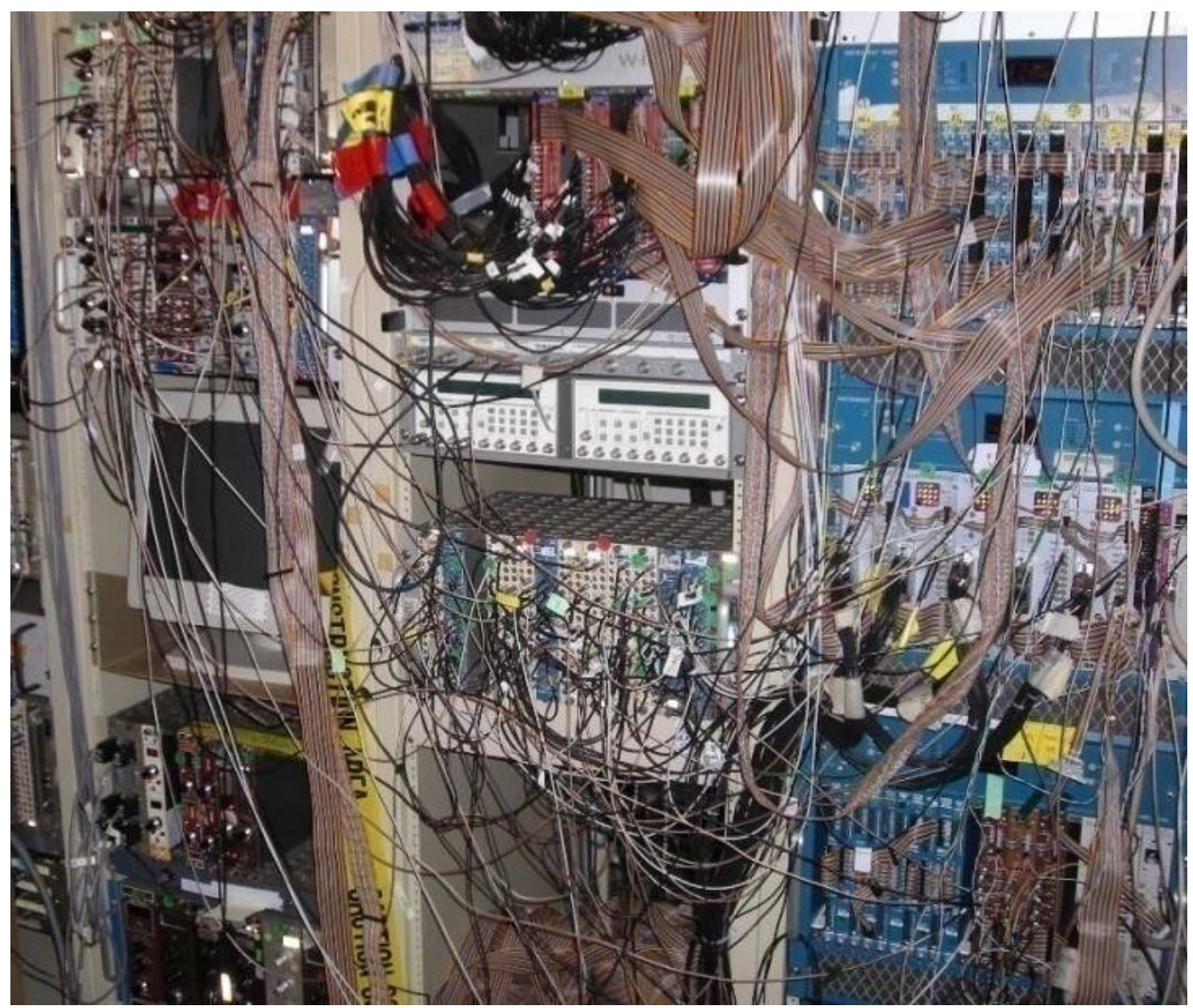

Figure 13. A portion of the detector electronics. These were used to create the data acquisition trigger and collection.

\subsection{Data Analysis Software}

The software used to analyze the data collected was gf3, a freely available gamma spectroscopy software package from Radware [35]. Gf3 is a powerful spectroscopy tool, with the ability to interactively display, perform peak searches, and carry out complicated fits to observed peaks of 1-dimensional spectra. The version of gf3 used for this analysis was modified by Mark Caprio of Yale University (currently a professor at Notre Dame) to read in matrices and perform gates on selected peaks [36].

Matrices are comprised of coincident events - if two (or more) events are detected simultaneously, the data is added to the $x$ - or $y$-axis of a matrix. The modified version of gf3 reads in 1-dimensional spectra as well as 2-dimensional 
matrices. A user can then choose to show an $\mathrm{X}$ or $\mathrm{Y}$ projection of the matrix. Upon viewing the projection, a gate can be set on a selected peak. A gate defines a region of interest for a coincidence. For example, a gamma-gamma coincidence matrix contains energy information of gamma rays in coincidence with one another. The coincidence is defined as occurring simultaneously. These are gamma rays that are emitted following a nuclear decay, when a cascade of gamma rays is emitted as the nucleus de-excites to the ground state. In gf $3 \mathrm{~m}$, a gate is set on a peak in the projection and all gammas that were coincident with the chosen peak will be displayed. An example of a gate in gf3m is shown in Figure 14, with the resulting coincident peaks in Figure 15.

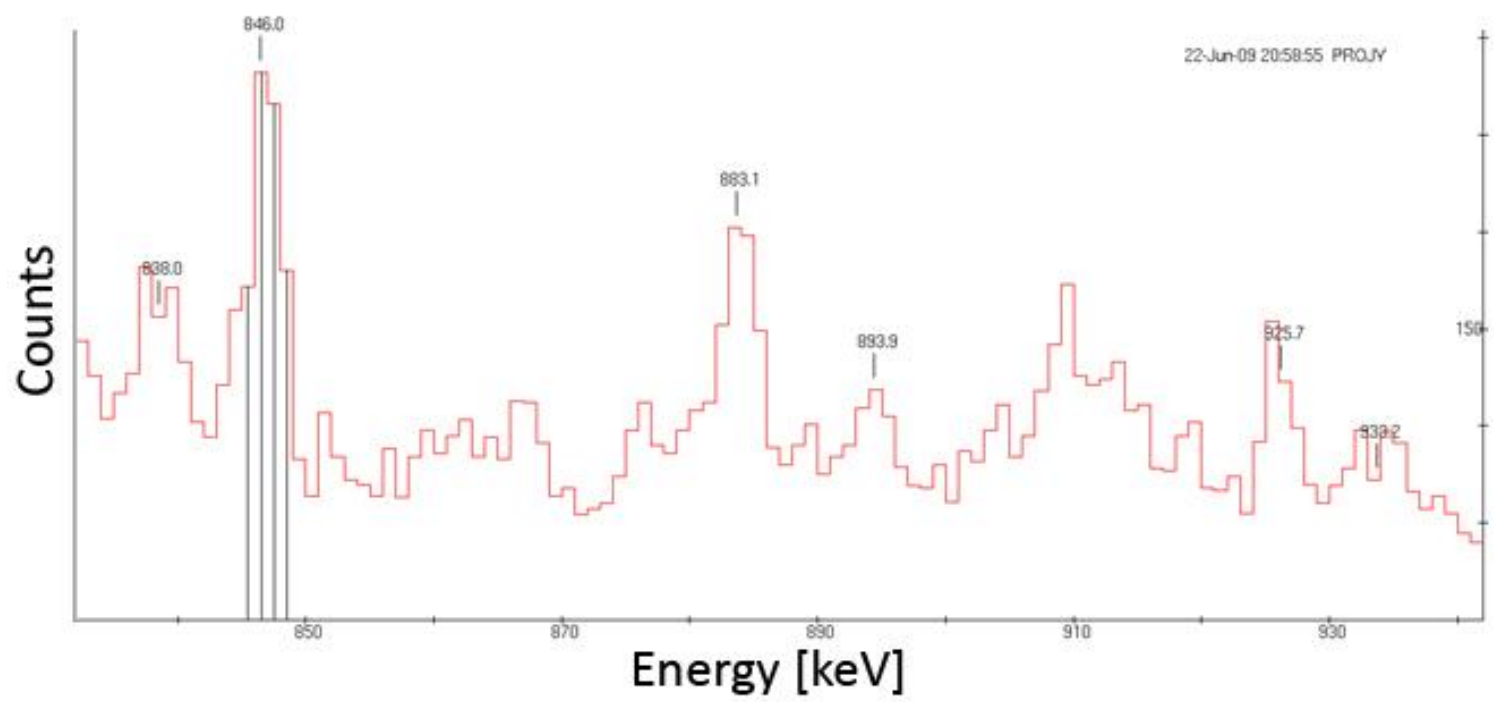

Figure 14. Screenshot of gate created on a peak of interest in gf3m. Background regions can also be selected for subtraction. 


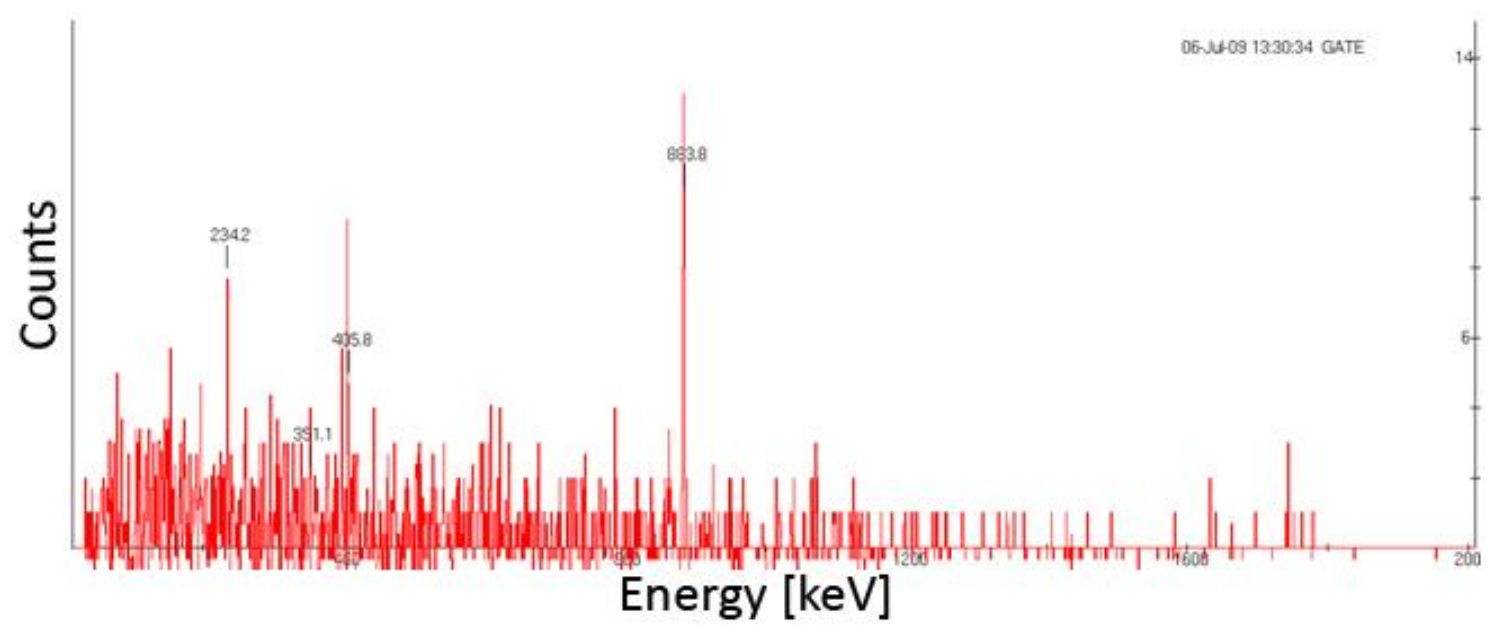

Figure 15. Peaks coincident with the gate shown in Figure 14.

To understand the population of isotopes in this experiment, the gammagamma coincident matrix was primarily used. Upon finding all the gamma energies coincident with the peaks of interest, these peaks were matched with public data from the NuDat 2.5 Levels and Gammas Search at Brookhaven National Laboratory [18].

\subsection{Times}

To see the delayed events, two clocks were used, a Time to Amplitude Converter TAC (TAC limit set to $\sim 50 \mu \mathrm{s}$ ) and the normal DAQ clock (Computer Automated Measurement And Control - CAMAC clock). The TAC has a start and stop signal - start was particle, stop was gamma or fission whereas the DAQ clock is just an oscillator, counting tics. When a particle is detected, the DAQ time is noted, and when a gamma or fission is detected, the new time is noted and it is determined how much time has elapsed. For example, if a "beam-off" 
and a gamma event are detected within the TAC limit, this event would have the TAC time assigned to it. If the event is detected outside of the TAC limit, it would be assigned the DAQ clock time. There is no limit on the DAQ clock, as long as 22 seconds is not surpassed without knowing it.

The TAC was necessary to fully capture all of the data, as the DAQ clock cannot measure events under $60 \mu \mathrm{s}$. When a particle is detected, the data acquisition system is busy processing the data for approximately $40-60 \mu \mathrm{s}$. During this time, if a gamma or fission is detected, the data cannot be collected as the data acquisition system is unavailable. To circumvent this loss of data, the TAC was added. If a particle is detected, the TAC is started. If, after $50 \mu \mathrm{s}$, a gamma or fission event is not detected, the TAC is cleared and the particle data is written to a file. If a gamma or fission event is detected within $50 \mu \mathrm{s}$ after the particle, the gamma/fission data, followed by the particle data, are written to a file. Figure 16 illustrates this process. 


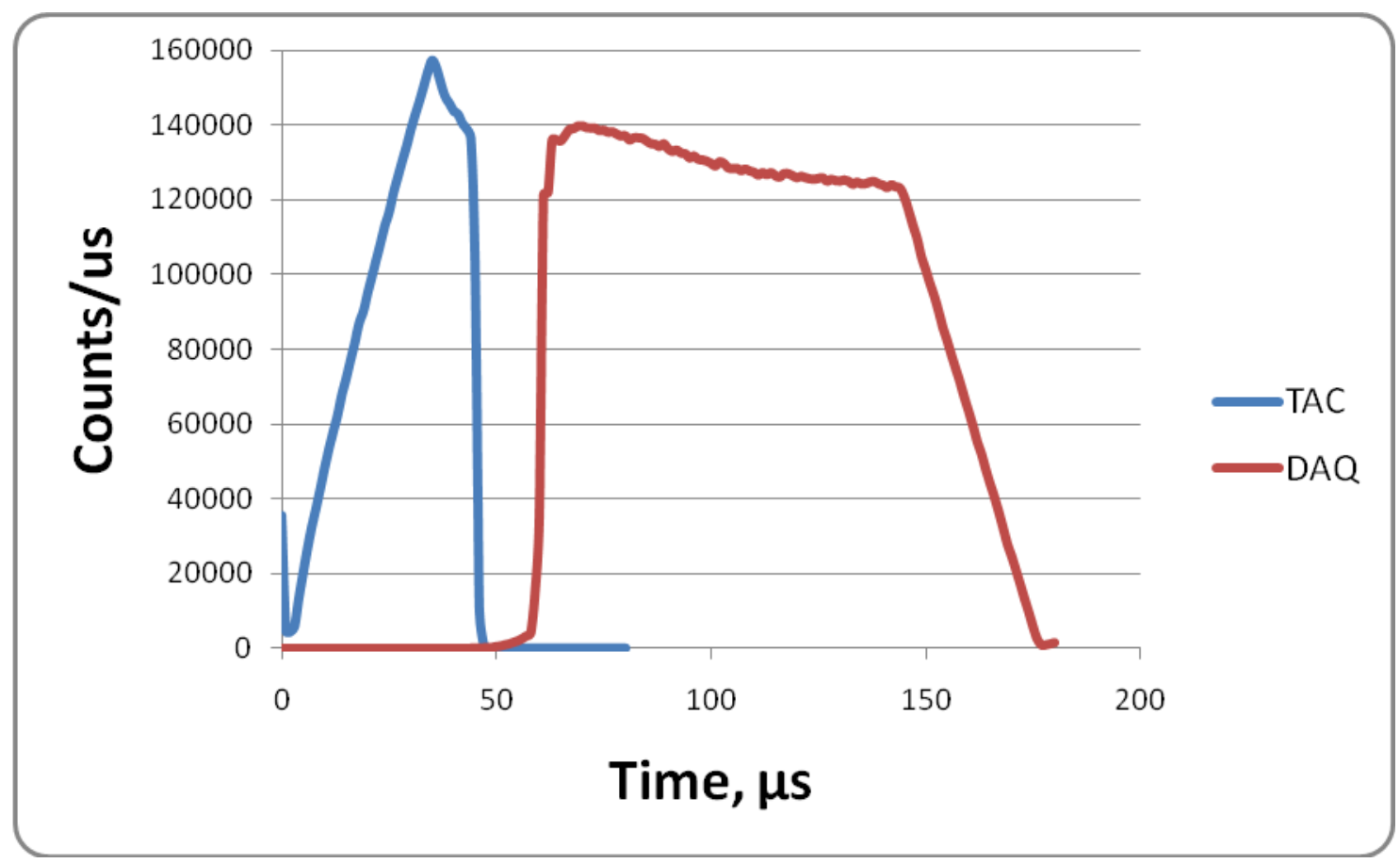

Figure 16. Counts assigned to the TAC and DAQ clocks. The clocks are started by a particle, and stopped by a gamma or fission event. The TAC clock captures events that occur before the DAQ is ready to record data. The counts drop off at $45 \mu \mathrm{s}$ as the DAQ is supposed begin recording. The DAQ is not able to begin recording until $\sim 55 \mu$ s after a particle is recorded.

The $10 \mu$ s gap between the end of the TAC and the beginning of the DAQ is attributed to an experimental error - the TAC was stopped too early.

\subsection{Data and Observations}

To gain meaningful information from the data collected, the Si and HPGe detectors must be time and energy calibrated. It was originally thought that each detector could be energy calibrated during several initial calibration runs; 
however, there was significant energy drift between many of the runs for the HPGe detectors. This caused every detector to be manually checked for drift after every run, with many of them requiring repeated calibrations. Within each Canberra germanium system, there are four crystals, each of which acts as a separate detector. This results in a total of 20 detectors all of which had to be calibrated across 51 runs, resulting in 1020 checks and potential calibrations.

Figure 17 shows a small example of the calibration checks. The numbers are intentionally illegible; the image is only intended to show the general trend. The green boxes illustrate data that lies within calibration, the yellow and red boxes are out of calibration. The top row is a sample of the detectors after using the initial calibration; the bottom row depicts the same sample upon calibration after each individual run.

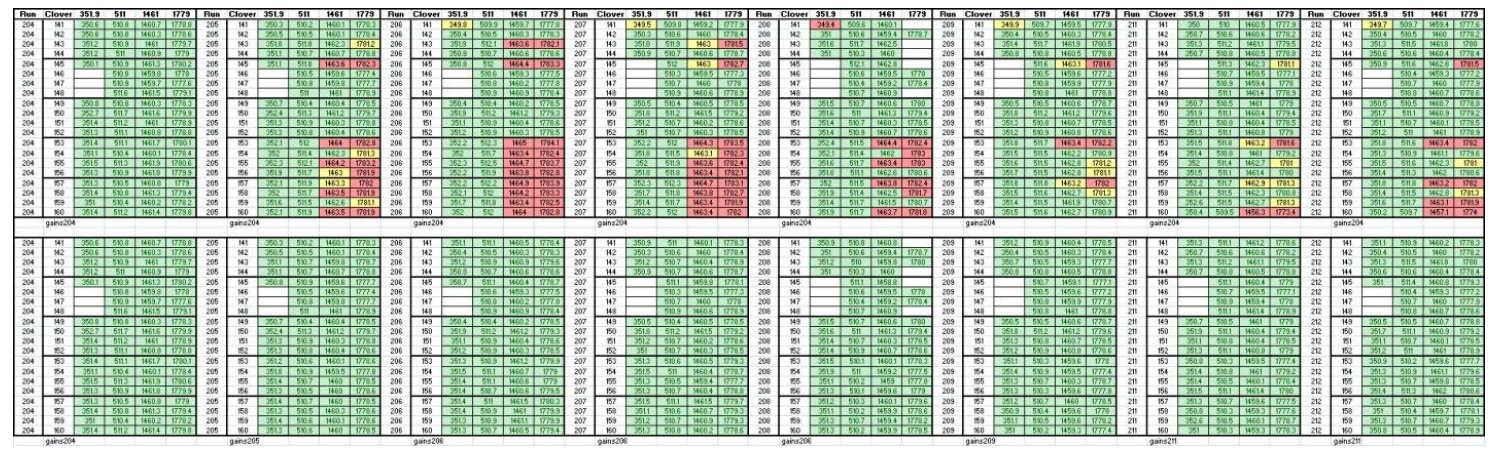

Figure 17. Small sample of the HPGe detector calibration trends. The $X$ axis represents calibration peaks and the $\mathrm{Y}$ axis represents individual detectors.

For the detector calibration, the following peaks were used:

$351 \mathrm{keV}-$ From the ${ }^{238} \mathrm{U}$ decay chain (due to a previous contaminant)

$511 \mathrm{keV}$ - Annihilation photons

$1460.8 \mathrm{keV}-{ }^{40} \mathrm{~K}$ in the concrete structure surrounding the experiment 
$1779 \mathrm{keV}$ - Aluminum/Silicon excitation in the equipment

These peaks were chosen due to their strong signal in all of the runs (with the exception of the $351 \mathrm{keV}$ gamma in the second clover, which is the cause of the blank boxes in Figure 16). Initially, a ${ }^{152}$ Eu calibration source was used. Those readings had to be discarded due to the drift issues discussed previously.

To correct for the drift between runs, a gain file was created for each run that held the calibration data. When histograms were created from the data provided by each run, the corresponding gain files were referenced resulting in correct peak alignment.

\subsection{Analysis}

Upon collection of the data from the detectors, the information was reviewed to determine if isomers were present. In order to obtain meaningful results from the data, the detectors were calibrated, and the gains were adjusted accordingly.

Data was sorted into various gamma histograms, including:

Gamma energy singles

Particle-gamma coincidences

Gamma-gamma coincidences

Gamma vs. TAC time

Gamma vs. DAQ time 
The histograms were created for each time window, with and without coincident fission events. The TAC time window was $5-45 \mu \mathrm{s}$, while the DAQ time window was 45 to $100 \mu \mathrm{s}$.

To search for isomers, the gamma-gamma coincidence data was read into the gf3m software as a two dimensional matrix consisting of isomeric gammas detected within $45 \mu$ s of a particle. A projection of the gamma-gamma matrix is shown in Figure 18. The large density of peaks makes spectroscopy a challenging endeavor.

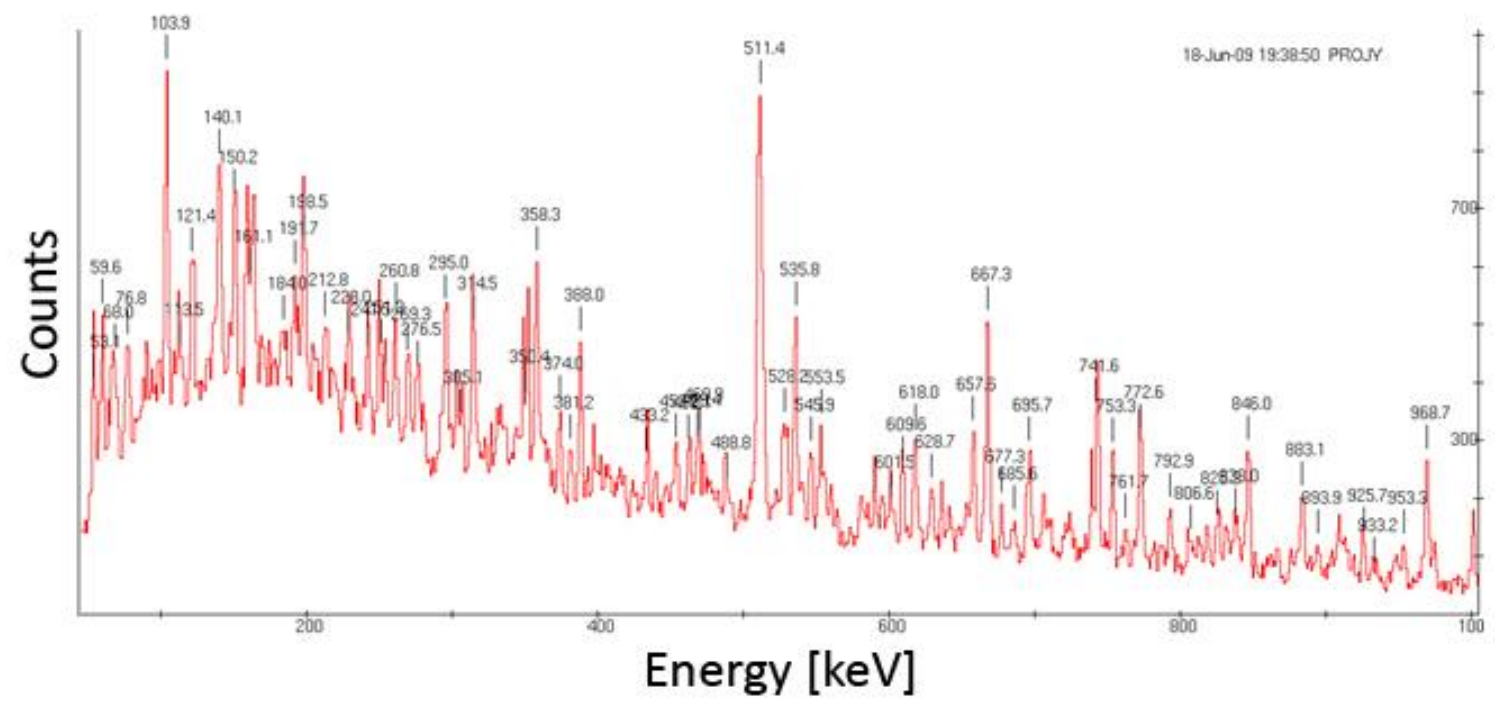

Figure 18. Y-axis projection of the gamma-gamma coincidence matrix in gf $3 \mathrm{~m}$. This projection is for gammas detected within $45 \mu$ s of particle.

After plotting the $y$-axis projection spectrum, all significant peaks above $100 \mathrm{keV}$ were recorded on the $\mathrm{x}$-axis of a spreadsheet. Peaks were deemed significant if they were at least 4 standard deviations above the background. Gf3m has a peak search algorithm that defines a peak relative to background. 
Users can define minimum sigma (number of standard deviations above background counts), full-width-at-half-maximum (FWHM, estimate of peak width), and percent area of the strongest peak found (relative strength to strongest line). In this work, a sigma value of $4, \mathrm{FWHM}$ of $5 \mathrm{keV}$, and percent area of $5 \%$ was used for the peak-fitting function of gf $3 \mathrm{~m}$. With a $4 \sigma$ confidence interval, the peaks picked out from the matrix projection were statistically significant. Peaks from known background radiation were ignored. Examples of these peaks would be the $511 \mathrm{keV}$ annihilation peak, or the $1460.8 \mathrm{keV}{ }^{40} \mathrm{~K}$ peak which was emitted by the surrounding concrete structure.

Once all the peaks of interest were catalogued, "gates" were systematically created on each one of the peaks. A gate is a region of interest in the $2 \mathrm{~d}$-histogram. When a gate is created, the matrix is read by the software to find all coincident peaks. Often times these peaks tend to be very small or not discernable from the background noise in the original projection. As the high and mid-level peaks are identified, the energies are recorded in the spreadsheet along the $y$-axis, under the corresponding energies from the original projection. Table 5 contains an example of this process. 
Table 5. Example of peak tracking method. The energies along the top $x$ axis were the prominent peaks in the original projections. Once a gate was created on the peak, the values on the $y$ axis represent coincident peaks that were displayed. Peaks marked blue belong to isomers, and nothing relevant was observed with those in black. ${ }^{134} \mathrm{Xe}$ is highlighted in green as the information on this isomer is used as an example.

\begin{tabular}{|c|c|c|c|c|c|c|c|c|c|c|c|}
\hline Energy [KeV] & 103.9 & 198.5 & 314.5 & 535.8 & 667.3 & 695.7 & 741.6 & 772.6 & 846 & 883.1 & 968.7 \\
\hline Isotopes & Sn-122 & Sn-120 & Ru-103 & Xe-132 & Xe-132 & Cs-124 & Ru-103 & Xe-132 & Xe-134 & Xe-134 & Sn-124 \\
\hline & & Xe-136 & (low conf) & & & (low conf) & (low conf) & & & & \\
\hline 91 & & 91 & & & & & & & & & \\
\hline 103.7 & & & & & & & & & & & 103.7 \\
\hline 151.7 & & & & & & 151.7 & & & & & \\
\hline 159 & & & & 159 & & & & & & & 160 \\
\hline 163.7 & 163.7 & & & & & & & & & & \\
\hline 197 & & & & & & & & & & & 197 \\
\hline 234 & & & & & & & & & 234 & & \\
\hline 242 & & & & & & & & 242 & & & \\
\hline 253 & & & & & & & & & & & 253 \\
\hline 314.8 & & & & & & & 314.8 & & & & \\
\hline 357 & & & & & & & & & & 357 & \\
\hline 381.5 & & 381.5 & & & & & & & & & \\
\hline 414 & & & & & 414 & 415 & & & & & \\
\hline 405 & & & & & & & & & 405 & & \\
\hline 526.7 & & & 526.7 & & & & 527 & & & & \\
\hline 535 & & & & & 535.9 & & & & & & \\
\hline 600 & & & & 600 & 600 & & & & & & \\
\hline 630 & & & & & 630 & & & & & & \\
\hline 667.6 & & & & 667.6 & & & & 667.4 & & & \\
\hline 696 & 696 & 697 & & & & & & & & & \\
\hline 739.1 & & & & 739.1 & 738.9 & & & & & & \\
\hline 740.6 & & & & & & & & 740.6 & & & \\
\hline 742.9 & & & 742.9 & & & & & & & & \\
\hline 753.6 & & & 753.6 & & & & 754.3 & & & & \\
\hline 772.2 & & & & & 772.2 & & & & & & \\
\hline 846.6 & & & & & & & & & & 846.6 & \\
\hline 884 & & & & & & & & & 883.3 & & \\
\hline 970 & 970 & & & & & & & & & & \\
\hline 974 & & & & & & 974 & & & & & \\
\hline 993 & & & & & & & & & & & 993 \\
\hline 1000.5 & 1000.5 & & & & & & & & & & \\
\hline 1023 & & 1023 & & & & & & & & & \\
\hline 1139.4 & 1139.4 & & & & & & & & & & \\
\hline 1171 & & 1171 & & & & & & & & & \\
\hline 1230.5 & & & & 1230.5 & & & & & & & \\
\hline 1314 & & 1314 & & & & & & & & & \\
\hline
\end{tabular}

Once all of the peaks are recorded from the gates made on the matrix projection, their origin is then sought. To determine the isotope of the peaks in the spreadsheet columns, NuDat 2.5 via the National Nuclear Data Center (NNDC) hosted by Brookhaven National Laboratory is used [18]. Upon entering NuDat 2.5, the Levels and Gammas search is selected, opening the Nuclear Levels and Gammas Search. The Nuclear Levels and Gammas Search allows one to search on many different parameters; however, the parameters of primary interest are the three gamma conditions (an upper and lower bound with the units of $\mathrm{keV}$ ), and an upper and lower bound assigned to $\mathrm{A}$, the atomic number. 
Bounding the atomic number is useful because some searches tended to yield a great number of results; removing radionuclides known to be out of bounds simplifies the identification. Since the fission fragments are distributed around the well-known and documented "double-hump" curve [7], nuclides above and below those peaks can be eliminated. The theoretical double-hump is shown in Figure 19. The shape of the fission fragment distribution curve was compared with the experimental data collected, displayed in Figure 20.

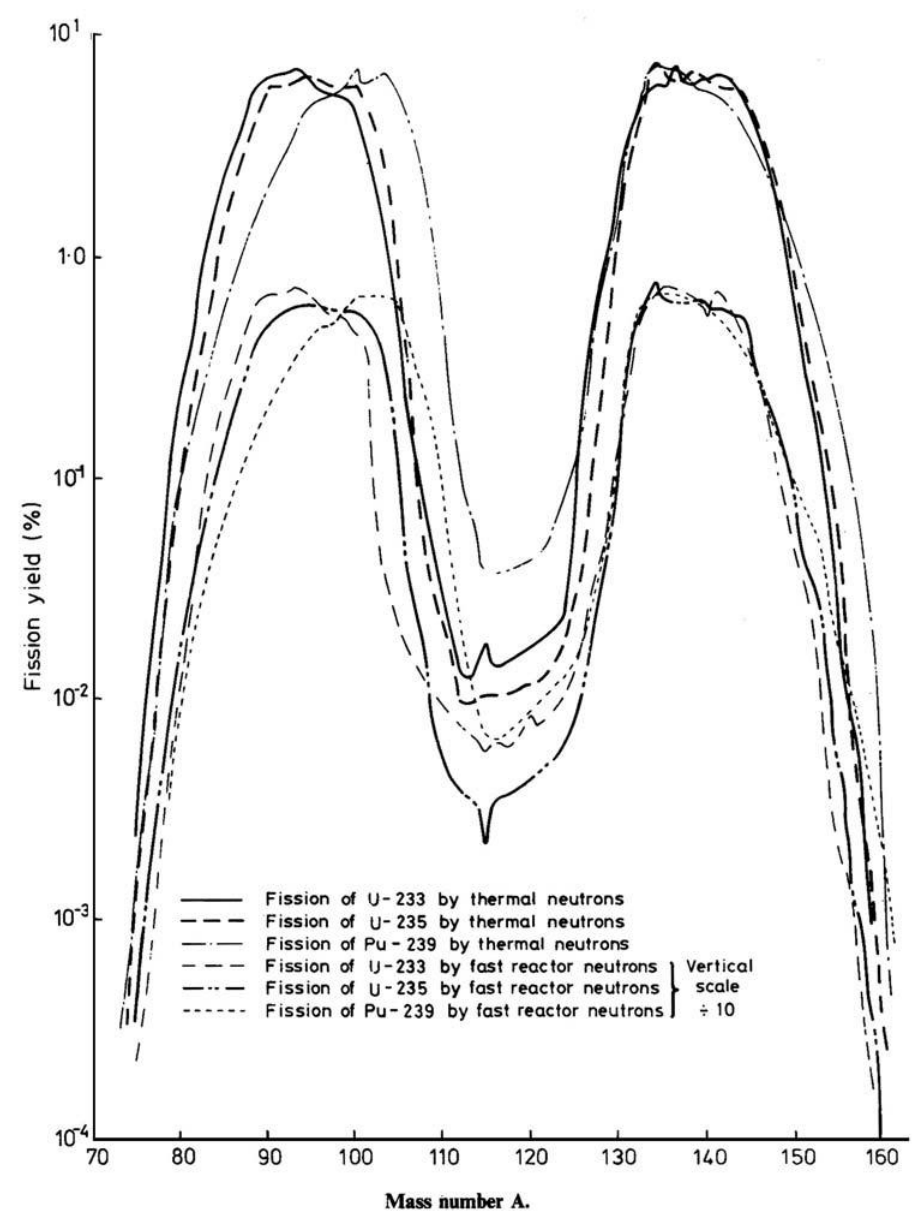

Figure 19. Theoretical fission fragment distribution 


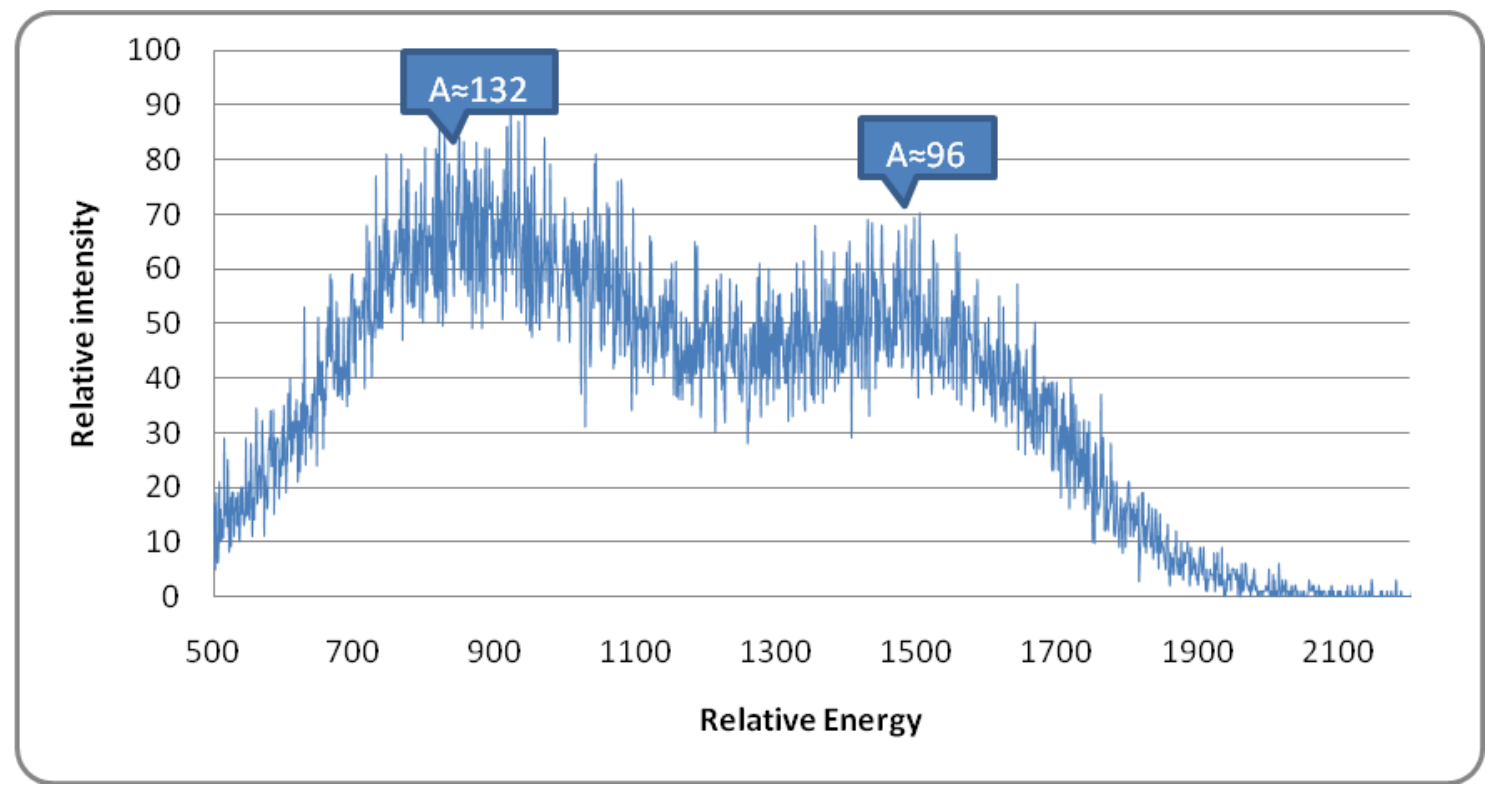

Figure 20. Experimental fission fragment distribution

It is worth noting that when comparing the theoretical distribution to the observed distribution, the curve representing the experimental data exhibits more symmetric fission. This can be attributed to the use of the lithium ions energized to $45 \mathrm{MeV}$ (9 MeV above the Coulomb barrier) inducing fission. The fission distribution for thermal neutrons, shown in Figure 18, shows low yields for the A 120 region. As higher energy neutrons are used, this yield increases. A similar effect is occurring here; the high-energy ${ }^{6} \mathrm{Li}$ ions are inducing symmetric fission.

Upon setting the constraints for the gamma ray energies and atomic number, a search is performed. The search typically yields many different radionuclides that have gamma rays within the specified ranges. The search was first narrowed by constraining the atomic number $A$. The second cut to narrow 
down the list is carried out by looking at $\mathrm{J}^{\Pi}$ (the spin number) and the excitation energy. Isomers typically do not have very large spin numbers so any entries fully populated with large spin numbers $( \pm 10)$ are not considered. If this assumption is invalid, the gammas emitted further down the decay chain will be seen and the isotope properly identified.

The list of remaining radionuclides is investigated individually, looking for traceable decay chains, where the isomer is releasing energy in discrete amounts to return to the ground state. This involves finding an observed energy, and tracing up and down the various energy levels to see if any other energies in the direct de-excitation chain are matched. If a full path can be traced with matching energies, and they all have a significant intensity (probability), the radionuclide is recorded as an observed isomer. Figure 21 shows an example based on the ${ }^{134} \mathrm{Xe}$ isomer, where two decay chains are present: with one starting at $1965.5 \mathrm{keV}$ and the other at $3025.2 \mathrm{keV}$. Both decay to the 1731.2 $\mathrm{keV}$ energy level, where they then emit 884.090 and $847.025 \mathrm{keV}$ gammas to get to the ground state. The gamma rays used to initially identify ${ }^{134} \mathrm{Xe}$ can be seen in Table 5. 


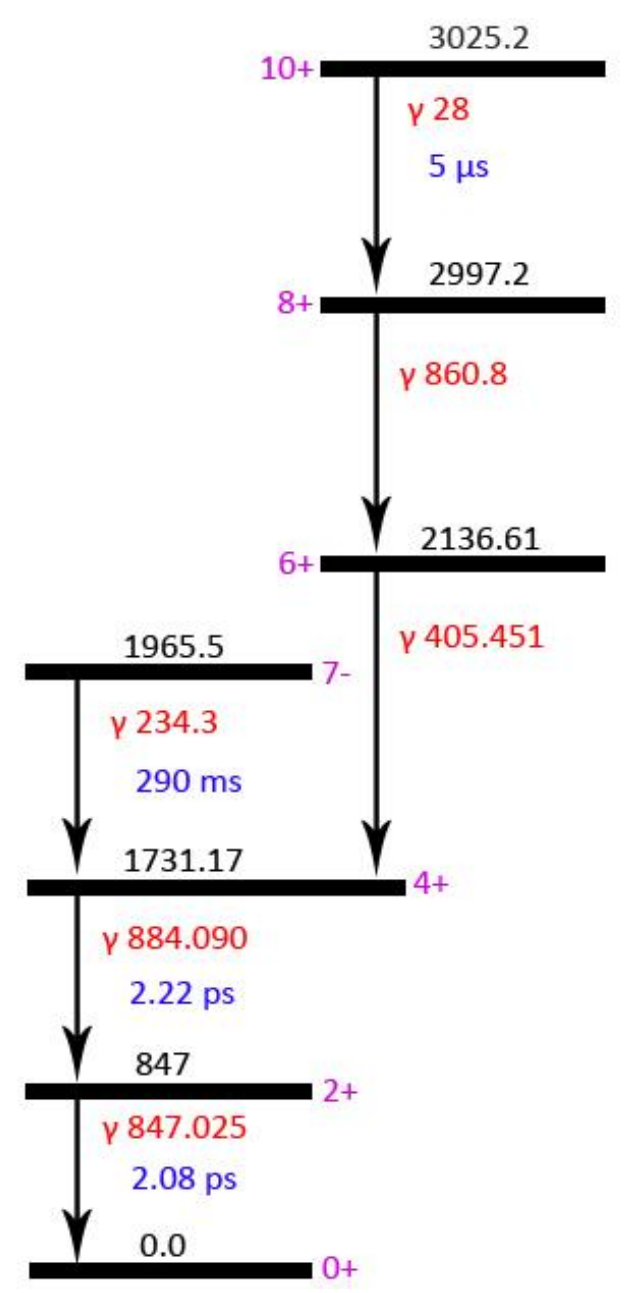

Figure 21. Isomeric decays in ${ }^{134} \mathrm{Xe}$

It is known that isomers are being seen as only delayed events are counted. While the beam is on and prompt gammas are being created, the detectors are not counting - this ensures that gammas recorded by the detector are delayed. In addition, two clocks (TAC and DAQ) are used to add timing information.

The times being recorded for the radiation interactions are useful in characterizing the isomers. For example, in gf $3 \mathrm{~m}$, one can choose to only display 
peaks created within a certain timeframe. It can be useful for analysis to view two different spectra at the same time, with each looking at a different window in time. For example, in Figure 22, the spectra with clock time 5-45 $\mu$ s (shown in red) and clock time $60-100 \mu s$ (shown in blue) are overlaid, making differences easy to visually recognize.

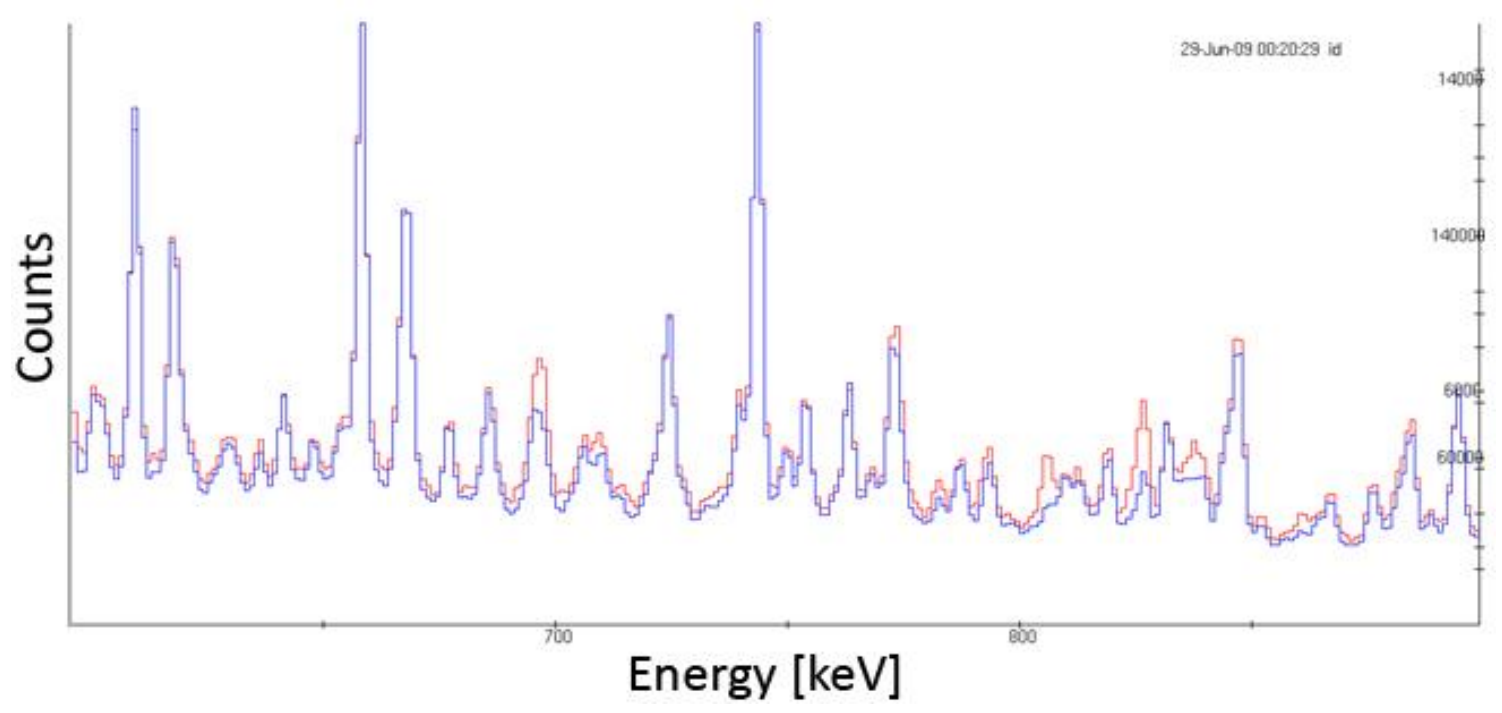

Figure 22. Gamma ray energies within $60-100 \mu$ s and $5-45 \mu$ s of detecting a particle. The blue spectrum belongs to the 60 - $100 \mu$ s counts and the red spectrum belongs to the 5-45 $\mu$ s counts. Short-lived isomers are only observed in the first time window (red), while background is observed in both.

These spectra can be subtracted to highlight only those decays that occur on a short timescale. This is done by subtracting the DAQ spectrum (60 - 100 $\mu \mathrm{s})$ from the TAC spectrum $(5-45 \mu \mathrm{s})$ to eliminate contaminants. There are a large amount of random correlations and contamination in both time spectra. These unwanted events are due contributions from the beta-decaying fission fragments (that build-up during the experiment), activation of the reaction 
chamber, and room background. As all time regions have these contaminants and random events, subtraction of one time region from another will effectively remove these contributions. The subtracted spectrum is shown below in Figure 23 , with peaks of isomers identified.

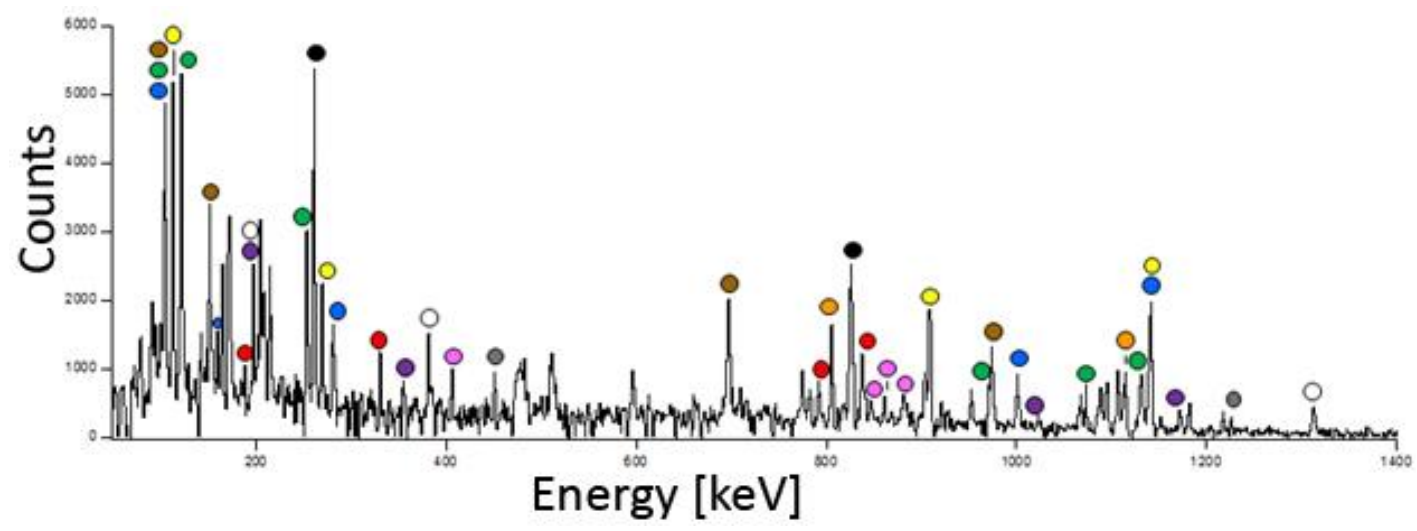

Figure 23. Decays recorded within $45 \mu$ s of a particle being detected. The colored circles represent gamma rays relevant to fission isomers, with the colors corresponding to the identified isomers listed in Table 6.

Table 6. Peaks identified from the spectrum shown in Figure 23.

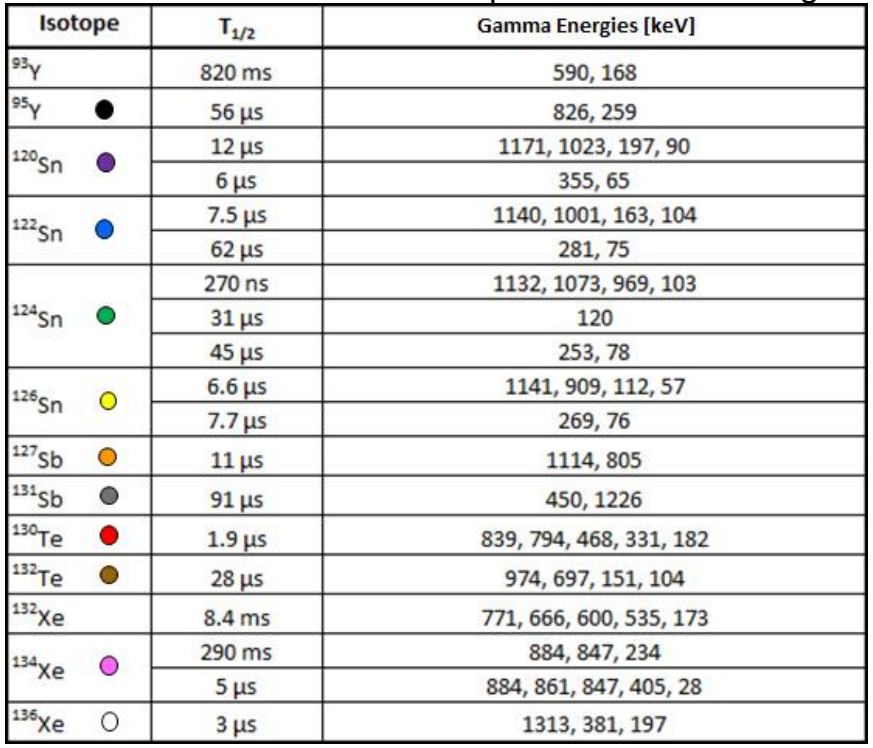


A wide range of isomers was observed in this experiment; a summary of short-lived isomers is documented in Table 6. Most of the isomers occur in the mass 95 and mass 135 regions, as expected, but also near mass 122 . The large population of these isomers is due to the symmetric fission observed, caused by the highly energized particle beam.

Note that ${ }^{93} \mathrm{Y}$ and ${ }^{132} \mathrm{Xe}$, with isomeric decay half-lives of $820 \mathrm{~ms}$ and 8.4 ms, respectively, are not observed in Figure 22. Due to the long half-lives, the peaks are removed in the time subtraction. These isotopes are strongly populated in the fission, and their absence in Figure 22 highlights the strength of the time subtraction. It is expected that this time selection and subtraction can be performed in an AID system out in the field to select short-lived isomeric decays.

The isomers characterized in Table 7 are relevant with respect to the goals of AID. One of the requirements was that the decay time must be less than $100 \mu \mathrm{s}$, as an interrogation beam is expected to be off for $20 \mathrm{~ms}$. All of the listed isomers meet this requirement besides ${ }^{93} \mathrm{Y}$ and ${ }^{132} \mathrm{Xe}$. The energies are desirable as well, most notably the tin isomers. These energetic photons are able to penetrate through more shielding than, for example, a $186 \mathrm{keV}$ gamma from ${ }^{235} \mathrm{U}$ decay. 


\subsection{Conclusions}

Analysis of the data created by this experiment shows that isomers from fissioning actinides can be detected and characterized. Since these isomers decay by emitting discrete energies with measurable unique decay times, it should be possible to determine which fission fragment is being detected using gamma spectroscopy. Ultimately, the only known elements that will undergo fission are those classified as special nuclear material (or other heavier manmade radionuclides), so the presence of certain fission fragment isomers is a clear indicator of their presence. Many of the isomer decay energies are over 1 $\mathrm{MeV}$ enabling them to penetrate shielding or surrounding cargo more effectively.

An example of this can be seen in the tin isomers, as all of them have gamma rays over $1 \mathrm{MeV}$. Isomers in ${ }^{120,122,124,126} \mathrm{Sn}$ were observed in this study. This is the first time these $\mathrm{Sn}$ isotopes have been studied using fission as a populating reaction $[18,37]$. These isomers have the potential to be useful in detecting SNM; however ${ }^{120} \mathrm{Sn},{ }^{122} \mathrm{Sn}$ and ${ }^{124} \mathrm{Sn}$ are naturally occurring stable isotopes with the potential to be inadvertently excited during an interrogation. However, ${ }^{126} \mathrm{Sn}$ is not stable, and has potential to be useful in AID. Another 
approach that can be investigated is looking at the ratios of tin isomers produced, as ${ }^{122} \mathrm{Sn}$ and ${ }^{124} \mathrm{Sn}$ only have a $4.63 \%$ and $5.79 \%$ atom percent abundance [11].

In addition to the potential national security applications via detector technology, this work shows the population of short-lived isomers in the A 122 region following fission. Furthermore, while there has been research on fissioning ${ }^{232} \mathrm{Th}$ with ${ }^{6} \mathrm{Li}$ [38], isomers from this reaction have not been characterized until now. This work expands the body of knowledge within fundamental nuclear physics, which is useful for researchers on future projects.

This work shows the achievement of isomer identification and observation with this experimental set-up. Search for the decay of the ${ }^{235} \mathrm{U}$ isomer is ongoing; however, the observation of the fission fragment isomers highlights the success of the technique. This work can be expanded to search for isomers in other forms of SNM such as ${ }^{239} \mathrm{Pu}$. The research performed for this thesis is a small part of the overall project. Future work for the Actinide Isomer Identification (AID) project includes continued research in an attempt to find isomers of the actinides themselves rather than just the fission fragments. The actinide isomers would be optimal because they would directly lead to identification of the actinide.

The population of isomers in the actinide isotopes, as well as the fission fragments, must be studied with a neutron beam. The use of the ${ }^{6} \mathrm{Li}$ beam described here provided clean characterization data, and the next step is to see if 
the isomers can be observed with a set-up that more closely resembles a fielded AID system. One outstanding question is whether the isomers observed in the A 122 region will be reasonably observed with neutron interrogation.

Upon characterizing the gamma peaks with the NNDC search, another possibility of future work would be to create software that would search out decay chains after the gamma energies were entered into a graphical user interface (GUI). The manual search for radionuclides was very tedious and time consuming. This task would lend itself very well to being automated by an individual with sufficient computer programming knowledge. This would be a worthwhile endeavor because this manual approach is currently employed by physicists working on numerous other projects.

In closing, isomers that have yet to be documented can be discovered in a repeatable fashion. Actinide isomer identification research is relevant because of the potential for new spectroscopic detectors to be developed utilizing the knowledge gleaned from it. Additionally, this research adds to the overall body of knowledge in the field of nuclear physics, which is desirable for its basic intrinsic value of learning more about the world we live in. 


\section{Appendix A}

[1] Foxwell J W. The Prospect of Nuclear and Biological Terrorism, Journal of Contingencies and Crisis Management 1997; 5 (2): 98-108.

[2] Shultis K J, Faw R E. Fundamentals of Nuclear Science and Engineering. Boca Raton (FL): CRC Press; 2002. 591 p. Second Edition

[3] Ellingsen S A. Safeguards Against Nuclear Terrorism: HEU vs Plutonium, Defense\&Security Analysis 2008; 24 (2): 129-146.

[4] United States Nuclear Regulatory Commission [homepage on the Internet]. Washington (DC): 2007 Feb. 12. [cited 2009 June 22]. Available from: http://www.nrc.gov/materials/sp-nucmaterials.html.

[5] Knoll G F. Radiation Detection and Measurement. Hoboken (NJ): John Wiley \& Sons, Inc.; 2000. 802 p. Third Edition

[6] Martin J E. Physics for Radiation Protection. Weinheim, Germany: WILEY$\mathrm{CHC}$ Verlag GmbH \& Co. KGaA; 2004. 713 p.

[7] Krane K S. Introductory Nuclear Physics. Hoboken (NJ): John Wiley \& Sons, Inc.; 1988. 845 p.

[8] Geelhood B D, Ely J H, Hansen R R, Kouzes R T, Schweppe J E, Warner R A. Overview of Portal Monitoring at Border Crossings, IEEE Xplore 2004; 513517.

[9] Bunn M. Preventing a Nuclear 9/11, Issues in Science and Technology 2005; 21 (2): 55-62.

[10] Frost R. Nuclear Terrorism Post-9/11: Assessing the Risks, Global Society 2004; 18 (4): 397-422. 
[11] Baum E M, Knox H D, Miller T R. Nuclides and Isotopes Chart of the Nuclides. Cincinnati $(\mathrm{OH})$ : Knolls Atomic Power Laboratory; 2002. 88 p. 16th Edition

[12] Sprinkle Jr J K, Christiansen A, Cole R, Collins M L, Hsue S T, Knepper P L, McKown T P, Siebelist R. Low-resolution Gamma-ray Measurements of Uranium Enrichment, Applied Radiation and Isotopes 1997; 48 (10-12): 1525-1528.

[13] RITA Research and Innovative Technology Administration [homepage on the Internet]. Washington (DC): Bureau of Transportation Statistics; 2008 Dec.. [cited 2009 June 22]. Available from:

http://www.transtats.bts.gov/Fields.asp?Table_ID=1358.

[14] Duderstadt J J, Hamilton L J. Nuclear Reactor Analysis. New York (NY): John Wiley \& Sons, Inc.; 1976. 650 p.

[15] Radiation Detection Center. New Detectors Could "Smell" Smuggled Nukes [homepage on the Internet]. Livermore (CA): Lawrence Livermore National Laboratory; 2004 May 3. [cited 2009 June 23]. Available from:

http://rdc.llnl.gov/denver.html.

[16] Warren G A, Caggiano J A, Miller E A, Bertozzi W, Klimenko A, Korbly S E, Ledoux R L, Park W H. Nuclear Resonance Fluorescence of ${ }^{235} \mathrm{U}$ above $3 \mathrm{MeV}$, IEEE Nuclear Science Symposium Conference Record 2007; 2047-2049.

[17] Ahle L. Global Security, Medical Isotopes, and Nuclear Science, AIP Conference Proceedings 2007; 947 (1): 9-16.

[18] Sonzogni A. National Nuclear Data Center [homepage on the Internet]. Upton (NY): Brookhaven National Laboratory; n.d. [cited 2009 June 23]. Available from: http://www.nndc.bnl.gov/nudat2/.

[19] Schirmer J, Gerl J, Habs D, Schwalm D. Gamma decay of the Superdeformed Shape Isomer in ${ }^{236} \mathrm{U}$, Physical Review Letters 1989; 63 (20): 2196-2199.

[20] Weigmann H, J P Theobald, Evaluation of fission barrier parameters from nearbarrier Evaluation of fission barrier parameters from near-barrier, Nucl. Phys. A187, 305; 1972.

[21] Wolf K L, J W Meadow, Measurements on the Fissioning Isomer ${ }^{238 m} \mathrm{U}$ with the (n, n') and (d, pn) Reactions, Bull. Am. Phys. Soc. 19, No.4, 595, KH1; 1974.

[22] Vorotnikov P E, V A Vukolov, E. A. Koltypin, Yu. D Molchanov, G A Otroschenko, Production of Fission Isomers in the Reaction ${ }^{238} \mathrm{U}\left(\mathrm{n}, \mathrm{n}^{\prime}\right)$, Proc. Conf. Neutron Physics, Kiev, Part 3, p.239; 1977.

[23] Dmitriev S V, G A Otroshchenko, S M Solovyev, Yield of Fissionable Isomers from Reactions ${ }^{234} U\left(n, n^{\prime}\right),{ }^{236} U\left(n, n^{\prime}\right)$, and ${ }^{238} U\left(n, n^{\prime}\right)$, Yad. Fiz. 38, 1394; 1983. 
[24] Makarenko V E, Yu. D. Molchanov, G A Otroshenko and G. B. Yankov, Ternary fission of neutron induced uranium fissioning isomers, Nucl. Phys. A502, 363; 1989.

[25] Kukushkin, I. A., V. E. Makarenko, Yu. D. Molchanov, G. A. Otroshchenko, G. B. Yankov, Energies of Long-Range Particles in Ternary Fission of the ${ }^{238} \mathrm{U}$ Spontaneously Fissioning Isomer, Yad. Fiz. 54, 8; 1991; Sov. J. Nucl. Phys. 54, 4; 1991.

[26] Browne E, J K Tuli, A=236 Mass Evaluation, Nucl. Data Sheets 107, 2649; 2006.

[27] Chukreev, F E, J E Makrarenko, M J Martin, A=238 Mass Evaluation, Nucl. Data Sheets 97, 129; 2002.

[28] Martin, M J, A=241 Mass Evaluation, Nucl. Data Sheets 106, 89; 2005.

[29] Singh A, R Zywina, R B Firestone, Table of Superdeformed Nuclear Bands and Fission Isomers, Nucl. Data Sheets 97, 241; 2002.

[30] Günther W, K Huber, U Kneissl, H Krieger, H Ries, H Ströher, W Wilke, H J Maier, Systematics of photonuclear yields and cross sections for plutonium and uranium fission isomers, Nucl. Phys. A350, 1; 1980.

[31] Oberstedt A, Oberstedt M, Gawrys M, Kornilov N Identification of a Shape Isomer in ${ }^{235} \mathrm{U}$, Physical Review Letters 2007; 99: 042502-1-042502-4.

[32] Migneco E, Russo G, De Leo R, Pantaleo A Fission Isomer of 237Npm, Physical Review C 1977; 16 (5): 1919-1923.

[33] Wolf K L, Unik J P. The Fissioning Isomer ${ }^{237} \mathrm{~Np}^{\mathrm{m}}$, Physics Letters 1973; 43B (1): $25-$ 26.

[34] Caggiano J A. Re: AID Beam Equation [electronic mail on the Internet]. Message to: Francy C J. 2009 June 10 [cited 2009 June 10]. [about 662,000 bytes].

[35] Radford D. RadWare [homepage on the Internet]. Oak Ridge (TN): Oak Ridge National Laboratory; 2000 May 15. [cited 2009 June 23]. Available from: http://radware.phy.ornl.gov/

[36] Ressler J. The ' $m$ ' in gf3m [homepage on the Internet]. New Haven (CT): A. W. Wright Nuclear Structure Laboratory; 2004 Jan.. [cited 2009 June 23]. Available from: http://wnsl.physics.yale.edu/cscan/otherstuff.html\#mmm.

[37] Tuli J. Evaluated Nuclear Structure Data File (ENSDF) [homepage on the Internet]. Upton (NY): Brookhaven National Laboratory; 2009 June 26. [cited 2009 July 1]. Available from: http://www.nndc.bnl.gov/ensdf/. 
[38] Itkis I M, Mulgin S I, and others. What can one learn about lithium breakup from the fission reaction of ${ }^{232} \mathrm{Th}\left({ }^{6} \mathrm{Li}, \mathrm{f}\right)$ at energies around the Coulomb barrier?, Physics Letters B 2006; 640: 23-27 


\section{Distribution}

No. of

Copies

3 Oregon State University

Corvallis, OR 97331

Dr Todd Palmer

Oregon State University Library

Department of Nuclear Engineering and Radiation Health Physics Library

Distr.1 


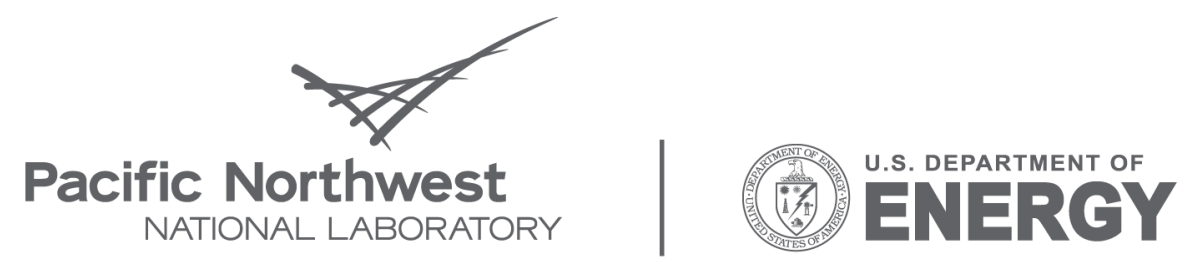

Proudly Operated by Battelle Since 1965

902 Battelle Boulevard

P.O. Box 999

Richland, WA 99352

1-888-375-PNNL (7665)

www.pnl.gov 